\title{
Altered Energy Metabolism During Early Optic Nerve Crush Injury: Implications of Warburg-Like Aerobic Glycolysis in Facilitating Retinal Ganglion Cell Survival
}

\author{
Jingyi Zhu ${ }^{1} \cdot$ Ping $\mathrm{Li}^{2} \cdot$ Yuan-Guo Zhou ${ }^{2} \cdot$ Jian $\mathrm{Ye}^{1}$
}

Received: 27 August 2019/Accepted: 28 October 2019/Published online: 10 April 2020

(C) The Author(s) 2020

\begin{abstract}
Neurons, especially axons, are metabolically demanding and energetically vulnerable during injury. However, the exact energy budget alterations that occur early after axon injury and the effects of these changes on neuronal survival remain unknown. Using a classic mouse model of optic nerve-crush injury, we found that traumatized optic nerves and retinas harbor the potential to mobilize two primary energetic machineries, glycolysis and oxidative phosphorylation, to satisfy the robustly increased adenosine triphosphate (ATP) demand. Further exploration of metabolic activation showed that mitochondrial oxidative phosphorylation was amplified over other pathways, which may lead to decreased retinal ganglion cell (RGC) survival despite its supplement to ATP production. Gene set enrichment analysis of a microarray (GSE32309) identified significant activation of oxidative phosphorylation in injured retinas from wild-type mice compared to those from mice with deletion of phosphatase and tensin homolog (PTEN), while PTEN-/- mice had more robust RGC survival. Therefore, we speculated that the
\end{abstract}

Electronic supplementary material The online version of this article (https://doi.org/10.1007/s12264-020-00490-x) contains supplementary material, which is available to authorized users.

Yuan-Guo Zhou

yuanguo.zhou@gmail.com

$\triangle$ Jian Ye

yejian1979@163.com

1 Department of Ophthalmology, Army Medical Center of the People's Liberation Army (PLA), Army Medical University, Chongqing 400042, China

2 Molecular Biology Center, State Key Laboratory of Trauma, Burn, and Combined Injury, Research Institute of Surgery, Army Medical Center of the PLA, Army Medical University, Chongqing 400042, China oxidation-favoring metabolic pattern after optic nervecrush injury could be adverse for RGC survival. After redirecting metabolic flux toward glycolysis (magnifying the Warburg effect) using the drug meclizine, we successfully increased RGC survival. Thus, we provide novel insights into a potential bioenergetics-based strategy for neuroprotection.

Keywords Optic nerve crush · ATP · Glycolysis · Oxidative phosphorylation $\cdot$ RGC survival

\section{Introduction}

Neuronal death is a major factor that causes permanent neurological disability after traumatic axonal injury, and the prevention of neuron loss has long been the focus of research on multiple fronts [1, 2]. In these research efforts, energy metabolism is a key factor, since central nervous system (CNS) tissues, particularly axons, are among the most metabolically demanding tissues $[3,4]$.

Early after CNS trauma, injured axons require extra energy to maintain subsequent energy-intensive processes, such as cytoskeletal structural rearrangements $[5,6]$. Energy deficits in axons have been reported to decrease neuronal function and survival [7]. Few attempts have been made to investigate the metabolic patterns of traumatized neurons and their translational significance for neuroprotection [8]. However, our understanding of energeticsbased pathological mechanisms in injured neurons is rather limited, hindering the development of bioenergetics-based treatments to address neuronal death [9]. Energy in the form of adenosine triphosphate (ATP) is generated by glucose metabolism in the CNS, predominantly through two main routes, glycolysis and oxidative phosphorylation. 
Each route has its own merits [10]; therefore, these routes are favored by cells in different states and serve diverse cellular processes. For instance, cancer cells and embryonic retinal cells prefer glycolysis and lactate metabolism even with abundant oxygen - this is also termed aerobic glycolysis or the Warburg effect [11-14]. However, the dependence of traumatized neurons on glycolysis and oxidative phosphorylation remains unclear [9]. A better understanding of the precise adaptation of energetic pathways to CNS trauma may be critical for providing novel insights into neuroprotection.

We therefore set out to investigate altered energy metabolism in injured neurons and determine its potential significance for neuronal survival early after trauma using the mouse optic nerve crush (ONC) model $[15,16]$.

\section{Materials and Methods}

\section{Animals}

All protocols were approved by the Administration of Affairs Concerning Experimental Animals Guidelines of the Third Military Medical University. Eight- to ten-weekold adult C57BL/6 male mice weighing $\sim 22 \mathrm{~g}-26 \mathrm{~g}$ were purchased from the Jackson Laboratory and housed in the Animal Care Center of the Research Institute of Surgery and Daping Hospital (Third Military Medical University, Chongqing, China). The mice were housed in groups of three under a 12-h light/dark cycle at room temperature (RT) of $22^{\circ} \mathrm{C}$ and given access to food and water ad libitum. All efforts were made to minimize animal suffering.

\section{Experimental Design and Statistical Analysis}

Physiological and biochemical indexes were measured on day 1 after surgery, unless otherwise noted, to evaluate the early-stage response.

\section{Surgical Procedures}

Mice were anesthetized via an intraperitoneal (i.p.) injection of pentobarbital sodium $(50 \mathrm{mg} / \mathrm{kg})$. The conjunctiva of the left eye was incised, and the orbital muscles were carefully moved aside to avoid damage to blood vessels. The exposed optic nerve was crushed $2 \mathrm{~mm}$ distal to the eyeball for $20 \mathrm{~s}$ using extra-fine self-closing forceps following previously published methods [16-18]. Proparacaine hydrochloride was applied during surgery and postoperatively as a local anesthetic. A sham operation was performed on the right eye as a self-control. All surgery was performed at 08:00, and tissue was harvested at the same time the next day, unless otherwise noted.

\section{Energy Metabolism Measurements}

ATP, ADP, and ADP/ATP Ratio Assays

The mice were sacrificed by cervical dislocation and the retinas and optic nerves were removed immediately. Approximately $6 \mathrm{~mm}$ of the optic nerve from the optic head containing the lesion was removed immediately and placed in lysis buffer (Cat. S0026, Beyotime, Chengdu, China) to halt metabolism. The tissue was dissected into small pieces and sonicated as described in a previous study [19]. The supernatant was collected after centrifugation at $12,000 \mathrm{rpm}$ for $10 \mathrm{~min}$ at $4^{\circ} \mathrm{C}$. The protein concentration was measured using a bicinchoninic acid protein assay kit (Cat. P0012, Beyotime). The supernatant was mixed with the prepared reagent and measured using a firefly luciferase-based ATP assay kit (Cat. S0026, Beyotime), an EnzyLight adenosine diphosphate (ADP) assay kit (Cat. EADP-100, BioAssay Systems, USA), and an EnzyLight $^{\mathrm{TM}}$ ADP/ATP Ratio assay kit (Cat. ELDT-100, BioAssay Systems) according to the manufacturers' instructions using a monochromator microplate reader (SafireII, Tecan, Switzerland). ATP concentrations were calculated from a log-log plot of the standard curve and normalized to the protein concentration.

\section{Lactate Assay}

Retinas were harvested and homogenized as described above. The lactate level in the supernatant was determined using a commercial colorimetric kit (Cat. AMEKO2677, Lianshuo Biological, China) according to the manufacturer's protocol.

\section{Lactate Dehydrogenase (LDH) Activity}

Supernatants of freshly dissected, homogenized optic nerves were obtained as described above. LDH activity in the samples was measured using an LDH assay kit (Cat. A020, Jiancheng, Nanjing, China) following the manufacturer's protocol.

\section{Staining Protocol}

\section{Retinal Whole-Mount Immunofluorescence Assay}

Retinal ganglion cell (RGC) survival was determined by counting the number of $\beta$-III-tubulin (Tuj1)-positive RGCs in retinal whole-mounts. Tuj1 specifically recognizes neuronal tubulin and is used as an RGC marker [20, 21]. The mice were anesthetized and transcardially perfused with $0.9 \%$ saline and $4 \%$ paraformaldehyde (PFA), after which the retinas were carefully harvested. Four 
symmetrical radial cuts were made on each retina. After postfixation in $4 \% \mathrm{PFA}$ for $1 \mathrm{~h}$ at $4^{\circ} \mathrm{C}$, the retinas were rinsed three times with phosphate-buffered saline (PBS) and simultaneously permeabilized and blocked in a solution of 3\% Triton X-100 in 10\% normal goat serum (Cat. ab7481, Abcam, Cambridge, UK) in PBS at room temperature. The samples were incubated with the primary antibody (1:600, Cat. ab78078, Abcam) for 2 days at $4{ }^{\circ} \mathrm{C}$. The retinas were washed three times with PBS for 15 min each and then incubated overnight with secondary antibodies [1:400, anti-mouse antibody conjugated with Alexa Fluor 488 (Cat. A-11001, Thermo Fisher Scientific, Waltham, MA, USA)] at $4^{\circ} \mathrm{C}$.

\section{Frozen Retinal and Optic Nerve Sections}

Eyeballs embedded in optimal cutting temperature compound were cut into $10-\mu \mathrm{m}$ sections along the sagittal axis on a freezing microtome. The middle portions of the optic nerves were cut into serial sections $8 \mu \mathrm{m}$ thick.

\section{Diaminobenzidine Cytochrome Oxidase Histochemistry}

Mitochondrial activity was investigated using diaminobenzidine cytochrome oxidase (DAB COX) histochemistry. $\mathrm{COX}$ is the terminal enzyme in oxidative phosphorylation and a reliable, well-established marker for measuring mitochondrial ATP generation. Frozen optic nerve sections were placed onto microscope slides and immersed. The DAB COX histochemistry procedure was conducted according to the manufacturer's protocol (Cat. Lt, R20440, Yuanye Biotechnology Co., Shanghai, China).

Five randomly-selected visual fields from each section proximal to the crush site were subsequently photographed. Brown or tan staining of the cytoplasm and/or membrane indicated positive labeling, and the mean optical density of brown or tan staining was analyzed.

\section{LDH Histochemistry}

A fresh slide of optic nerve was obtained as described above, and LDH was stained following the manufacturer's protocol (Cat. G2362, Solarbio, Beijing, China). Blue/ purple staining indicated positive labeling. Histochemical staining was automatically categorized as strongly positive, positive, weakly positive, or negative; then the number of positive cells was counted, and the samples were rated by ImageJ IHC Profiler (https://sourceforge.net/projects/ihc profiler/) as listed in Tables S1 and S2 in the Supplementary materials.
Image Acquisition and Analysis

All immunofluorescence images were acquired on an SP8 confocal microscope (Leica, Germany), and immunohistochemistry images were captured using a camera (Dfc290, Leica) attached to an upright microscope (Dm1000, Leica Microsystems, Germany).

To obtain RGC counts in retinal whole-mounts, eight fields from each retinal explant were randomly sampled at central and peripheral locations with progressive eccentricity from the optic nerve head through the midline of the four retinal quadrants at distances of 1.0 and $1.5 \mathrm{~mm}$ from the margin of the optic disc. Tuj1-positive RGCs in all fields were counted with ImageJ software (developed by Wayne Rasband, National Institutes of Health, Bethesda, MD; available at http://rsb.info.nih.gov/ij/index.html). The counts were averaged for each explant.

\section{Calculation of Changes in Glycolysis and Respira- tion Rates}

Three types of unilateral optic nerve crush (ONC) models were adopted; saline, carbonyl cyanide 3-chlorophenylhydrazone (CCCP), or 2-deoxyglucose (2DG) was injected 1 $\mathrm{h}$ prior to retinal extraction in each group. Then, ATP levels were measured in the following: the contralateral intact optic nerve $(\mathrm{CON})$, the injured optic nerve (ONC), the contralateral intact optic nerve after 2DG treatment $\left(\mathrm{CON}_{2 \mathrm{DG}}\right)$, and the injured optic nerve after 2DG treatment $\left(\mathrm{ONC}_{2 \mathrm{DG}}\right)$. The difference between $\mathrm{CON}$ and $\mathrm{CON}_{2 \mathrm{DG}}$ is the energy produced by glycolysis in the intact optic nerve $\left(\mathrm{E}_{2 \mathrm{DG} / \mathrm{C}}\right)$, and the difference between $\mathrm{ONC}$ and $\mathrm{ONC}_{2 \mathrm{DG}}$ is the energy produced by glycolysis in the injured optic nerve $\left(\mathrm{E}_{2 \mathrm{DG} / \mathrm{O}}\right)$. The ONC/CON ratio was a fixed cutoff point and the $\mathrm{ONC}_{2 \mathrm{DG}} / \mathrm{CON}_{2 \mathrm{DG}}$ and $\mathrm{ONC}_{\mathrm{CCCP}} / \mathrm{CON}_{\mathrm{CCCP}}$ ratios were compared to determine if the glycolysis and respiration rates increased or decreased. The specific calculations are shown in Fig. 5A.

\section{Reverse Transcription qPCR (RT-qPCR)}

TRIzol reagent was used for RNA extraction according to the manufacturer's instructions. The total RNA concentrations in the samples were measured using a NanoDrop system (Thermo Scientific). Then, the RNA was reversetranscribed using a reverse transcription kit (Cat. RR037A, TaKaRa Bio Inc.). Gene expression was quantified using a real-time fluorescence-based quantitative PCR kit (Cat. RR039B, TaKaRa Bio Inc.). Beta-actin was used as an internal control to normalize the RT-qPCR readout. 


\section{Flow Cytometry}

\section{Thy-1-Positive RGC Counting}

A previously reported flow cytometry-based Thy-1-labeling RGC counting method was also applied to assess RGC survival rates. RGCs were isolated using the method described by Chintalapudi [22, 23], with some modifications. Suspensions of retinal cells were generated using enzymatic digestion and subsequently filtered through a sterile $70-\mu \mathrm{m}$ nylon strainer to obtain single-cell suspensions. Each suspension was centrifuged for $10 \mathrm{~min}$ at 200 $\times \mathrm{g}$, and the supernatant was discarded. The pellet was resuspended in PBS/1\% FBS, and the cell density was determined. Two microliters of rat anti-mouse Thy-1.2 phycoerythrin (PE) (BD Biosciences, Cat. 553014) antibody were used to stain $\sim 5.0 \times 10^{6}$ cells in a $1000 \mu \mathrm{L}$ volume for $6 \mathrm{~min}$ at RT. The samples were analyzed using an ACEA NovoCyte instrument.

\section{Mitochondrial Labeling}

Mitochondrial function was evaluated with mitochondrial membrane potential-dependent $(\Delta \psi \mathrm{m})$ MitoTracker Deep Red FM staining. The cells were incubated with $100 \mathrm{nmol} /$ L MitoTracker Deep Red FM for $8 \mathrm{~min}$ at RT. Deep red fluorescence (excitation at $644 \mathrm{~nm}$, emission at $665 \mathrm{~nm}$ ) was detected through the allophycocyanin channel.

\section{Derivation and Analysis of Microarray Data}

We used the gene set enrichment analysis (GSEA) computational method to analyze results in the form of pathway rankings that indicated which pathways play important roles in injured wild-type mice compared to PTEN-deficient mice [24, 25]. The GSE32309 gene microarray data were downloaded from the Gene Expression Omnibus database [26] (http://www.ncbi.nlm.nih.gov/ geo/), which included three normal mice (GSM800486, GSM800487, and GSM800488) and three mice with PTEN deletion (GSM800489, GSM8004870, and GSM8004871) based on the Mouse Gene 1.0 ST Array from Affymetrix, Inc. (Santa Clara, CA, USA) [27]. The original CEL files were converted into expression measures and normalized using the affy package in the R language (http://www.bio conductor.org/packages/3.0/bioc/) [28]. Then, the data were analyzed with GSEA-3.0 software with the default parameters, except for the permutation parameter selection, which was set to "geneset" instead of "phenotype" [25]. The enrichment score, normalized enrichment score, $P$ value, and false discovery rate $\mathrm{Q}$ value were obtained from the GSEA output reports, and these were then used to rank the gene sets.

\section{Drug Administration and Effectiveness}

Doses of $500 \mathrm{mg} / \mathrm{kg}$ 2DG (D8375, Sigma-Aldrich), $4 \mathrm{mg} /$ $\mathrm{kg}$ CCCP (C2759, Sigma-Aldrich), $100 \mathrm{mg} / \mathrm{kg}$ meclizine (B1786, Apexbio), and $50 \mathrm{mg} / \mathrm{kg}$ or $5 \mu \mathrm{mol} / \mathrm{kg}$ ATP (PV3227, Thermo Fisher) were injected intraperitoneally.

We injected $500 \mathrm{mg} / \mathrm{kg} 2 \mathrm{DG}$ or $4 \mathrm{mg} / \mathrm{kg}$ CCCP immediately after surgery to investigate the numbers of surviving RGCs after inhibition of glycolysis and oxidative phosphorylation. For the analysis of glucose metabolism (Fig. 5), mice were injected with the same dose of 2DG $(500 \mathrm{mg} / \mathrm{kg}) /$ iodoacetic acid $(60 \mathrm{mg} / \mathrm{kg}) /$ oligomycin $(0.5$ $\mathrm{mg} / \mathrm{kg}) / \mathrm{CCCP}(4 \mathrm{mg} / \mathrm{kg}) 1 \mathrm{~h}$ prior to euthanasia.

\section{Statistical Methods}

The experimenter was blinded to the treatment of the mice when collecting and analyzing the data. The data are presented as the mean $\pm \mathrm{SD}$, and the error bars indicate the $95 \%$ confidence intervals. Paired $t$-tests were applied to compare eyes from the same animal or the optic nerve and retina from the same eye, and independent-samples $t$-tests were used for unrelated samples. Multiple comparisons were performed using one-way analysis of variance (ANOVA) followed by Tukey's or Dunnett's post hoc test, or two-way ANOVA followed by Sidak's post hoc test. Correlations were analyzed using the Pearson correlation test as appropriate. A $P$ value $<0.05$ indicated statistical significance.

Statistical analyses were performed using Sigma Plot version 13.0 and PRISM version 6.0 (GraphPad Software). Graphics were plotted in PRISM version 6.0 and Adobe Illustrator [29, 30].

\section{Results \\ ONC Triggers Metabolic Activation in Injured Optic Nerves and Retinas}

In this study, we used the ONC model to investigate the role of the energy budget in the pathology of acute traumatic axon injury (Fig. 1A). ONC is a classic animal model of neurodegeneration, with early axon loss and cell death 1 day after injury $[31,32]$. The visual system ranks among the most energy-demanding systems in the brain [33]. Consistently, our results showed that the retina had a much higher ATP content than the cortex and hippocampus in mature C57BL/6 mice at baseline (Fig. 1B).

We first measured the energy levels of retinas and corresponding optic nerves and found that the ATP levels were moderately increased after ONC (Fig. 1C). Their ADP levels also increased (Fig. 1D), supporting the 
A

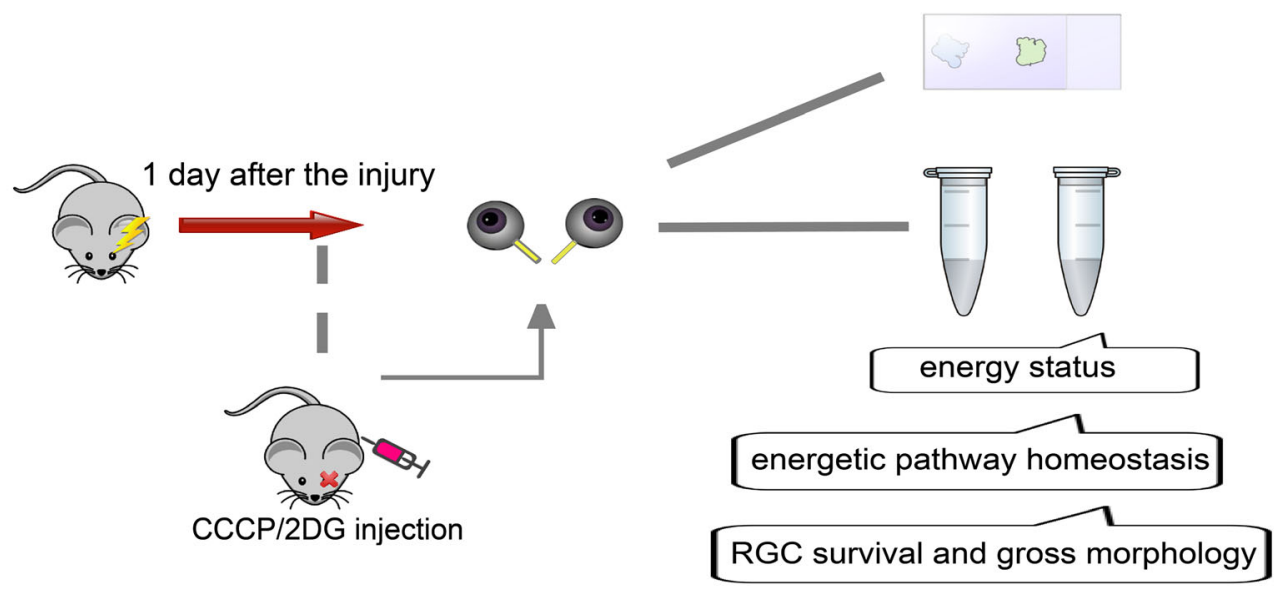

B

E
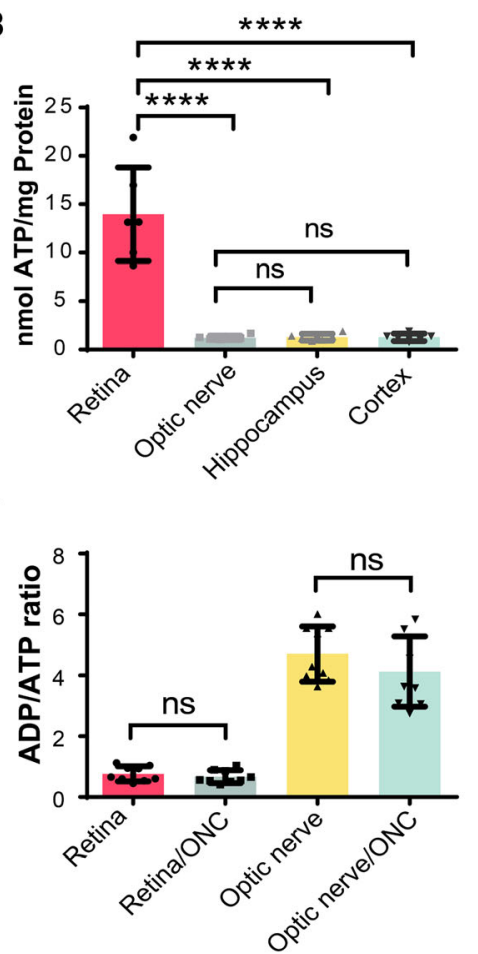

C

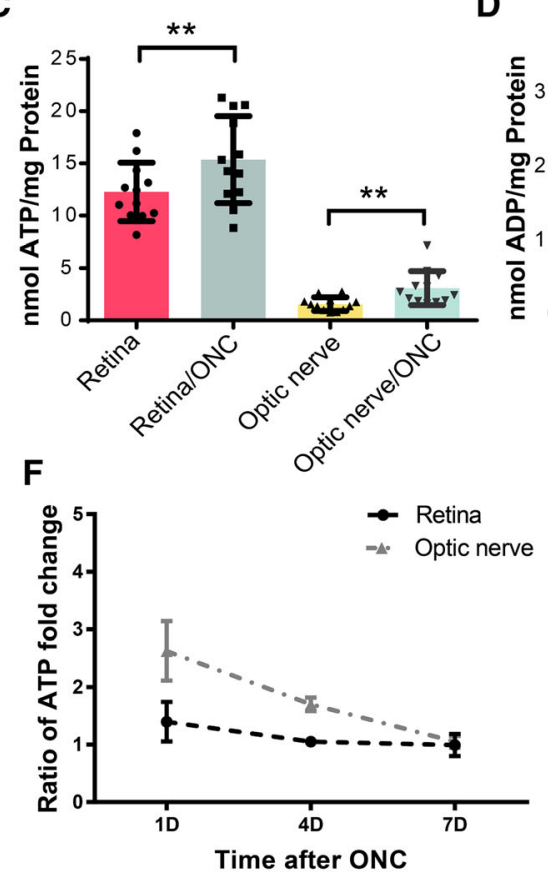

D

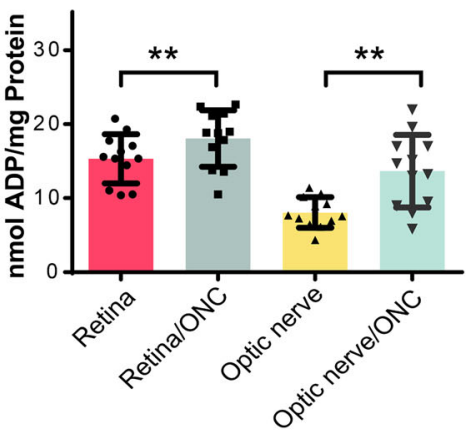

Fig. 1 Optic nerve crush (ONC)-stimulated energy metabolism in the retina and optic nerve. A Schematic of the experimental design. The main outcome measurements included the energy status (ATP levels, ADP levels, and the ADP/ATP ratio), energetic pathway homeostasis (glycolysis and oxidative phosphorylation activity and relative proportions), and retinal ganglion cell (RGC) status (numbers of surviving cells and gross morphology). B Basal ATP levels in the

hypothesis that neurons rapidly undergo a series of energyexpensive processes in response to injury [5]. The ATP and ADP increases were smaller in retinas than in optic nerves, probably because the nerves were directly traumatized, while only a small portion of the retinal cells (RGCs) was affected. However, the ADP/ATP ratio, a critical parameter of energy consumption and balance [34], showed no decrease, indicating that the relative energy equilibrium retina, optic nerve, hippocampus, and cortex. C, D ATP and ADP levels in retina and optic nerve 1 day after ONC. E The dynamic balance of energy metabolism presented as the ADP/ATP ratio. F ATP concentration-time curves for retinas and optic nerves 1, 4, and 7 days after ONC. $* * P<0.01, * * * * P<0.0001$; ns, not significant.

was maintained through robust energy consumption and generation (Fig. 1E).

We confirmed the stimulation of energy metabolism in the ONC model. Notably, when monitoring ATP concentrations for a longer period ( 3 and 7 days after injury) (Fig. 1F), this increase was not apparent in the retina but still occurred in the optic nerve after 3 days. Subsequently, this trend disappeared in both locations 7 days after injury. 
We further evaluated the activity of the two main glucose metabolism pathways in the visual system, glycolysis and mitochondrial oxidative phosphorylation. To assess mitochondrial activity in RGCs, we used a previously-reported flow cytometry-based assay. Isolated retinal cells were co-stained with MitoTracker Deep Red, a $\Delta \psi \mathrm{m}$ dependent dye that binds to metabolically active mitochondria, and Thy-1.2, an RGC-specific marker. The mean fluorescence intensity of MitoTracker Deep Red staining in Thy-1.2-positive cells indicated that the number of active mitochondria in RGCs increased after ONC (Fig. 2A). We then evaluated mitochondrial $\mathrm{COX}$ activity in the optic nerve using DAB staining. A substantial increase in mitochondrial staining was observed in the injured nerve (Fig. 2B). Moreover, the mRNA levels of tricarboxylic acid cycle enzymes (citrate synthase, $\boldsymbol{\alpha}$-ketoglutarate dehydrogenase, fumarate hydratase, and isocitrate dehydrogenase 1) were increased in the nerve (Fig. 2C). Based on these results, mitochondrial oxidative phosphorylation is enhanced in the ONC model.

The level of glycolysis is indicated by the expression and activity of the glycolytic enzyme LDH, a key enzyme in anaerobic respiration that catalyzes the final step of glycolysis by converting pyruvate to lactate. Quantitative analyses of LDH activity in tissue homogenates revealed a slight increase in injured optic nerve, but this activity remained basically unchanged in the whole retina (Fig. 2D). Qualitative analyses of LDH histochemistry also showed a higher level of staining in injured optic nerve (Fig. 2E and Table S1). The ganglion cell layer of the retina also displayed intense $\mathrm{LDH}$ staining in the ONC group, but no significant changes were evident in the rest of the retina (Fig. 2E). Thus, the LDH increase in RGCs may be masked by the remaining part of the retina, as shown in Fig. 2D. qRT-PCR revealed increased expression of the glycolysis enzymes hexokinase 1 and 2, phosphofructokinase-1, glyceraldehyde 3-phosphate dehydrogenase, pyruvate kinase, and lactate dehydrogenase $\mathrm{A}$ in optic nerve following ONC (Fig. 2F). Together, these findings indicate a trend of increased glycolysis in injured optic nerve.

\section{Neither Systemic Increases in Internal Energy Levels nor Supplementation with Exogenous ATP Improves RGC Survival}

What is the biological significance of this energetic activation? Since neurons are metabolically demanding and energy-dependent, we suspected that this post-trauma metabolic response was essential for RGCs to avoid energy deficits and survive. To test this hypothesis, we intraperitoneally injected mice with $2 \mathrm{DG}$, an inhibitor of the glycolytic enzyme hexokinase, and CCCP, a protonophore $\left(\mathrm{H}^{+}\right.$ionophore) and uncoupler of mitochondrial oxidative phosphorylation, immediately after surgery to restrict glycolysis or oxidative phosphorylation, respectively. The numbers of surviving RGCs were counted in Tuj1-labeled retina whole-mount preparations and using the flow cytometry-based Thy-1.2-labeling RGC sorting method. CCCP and 2DG significantly reduced RGC survival in the ONC model at 1 day; however, there were no significant effects on the intact retina (Fig. 3A, B).

Since blocking intense energy production is detrimental to RGC survival, does a larger energy reserve help protect against RGC loss? In other words, does a mouse with greater internal energy levels after injury have an advantage in terms of RGC survival? To determine the inherent energy potential of each mouse after injury, the ADP/ATP ratios of the injured and intact optic nerves from the same mouse were calculated. Pearson correlation analyses and regression analyses were used to examine the relationship between RGC survival and the fold change in the ADP/ ATP ratio (Fig. 4A). Surprisingly, no significant correlation was found ( $n=25 ; r=-0.2507 ; P=0.2268)$. Therefore, mice with a more energetically active optic nerve after injury have no advantage in terms of reduced RGC death.

Energy supply tends to promote neuronal survival following CNS injury [35, 36]. Given that more internal energy failed to produce more surviving RGCs, we questioned whether supplying extrinsic ATP would confer any benefit for RGC survival. ATP was injected at a relatively high dose $(50 \mathrm{mg} / \mathrm{kg})[37,38]$ and a low dose $(5$ $\mu \mathrm{mol} / \mathrm{kg} ; \sim 2.865 \mathrm{mg} / \mathrm{kg}$ ) [39]. Intraperitoneal injections did not have a significant effect on RGC survival in either injured or intact eyes (Fig. 4B, C).

\section{Energetic Reliance Shifts to Mitochondrial Respi- ration in Injured Optic Nerve}

Following our observation that both glycolysis and mitochondrial oxidative phosphorylation are upregulated after ONC, we questioned how strongly each pathway became elevated and contributed to ATP production. We established three groups of unilateral ONC models and measured the ATP content in the corresponding optic nerves (the meaning of each value is explained in the Methods). CCCP and 2DG were injected to inhibit respiration and glycolysis, respectively, into ONC mice, while saline was injected into control mice. Both crushed and sham-operated optic nerves were extracted $1 \mathrm{~h}$ after the injection, and the ATP levels were measured. According to the formula shown in Fig. 5A, the ATP ONC/CON ratios were set as fixed cutoff points to differentiate changes in the proportions of the different pathways after injury, and the $\mathrm{ONC}_{2 \mathrm{DG}} / \mathrm{CON}_{2 \mathrm{DG}}$ and $\mathrm{ONC}_{\mathrm{CCCP}} / \mathrm{CON}_{\mathrm{CCCP}}$ ratios were compared to the $\mathrm{ONC} / \mathrm{CON}$ value to determine if the rates 

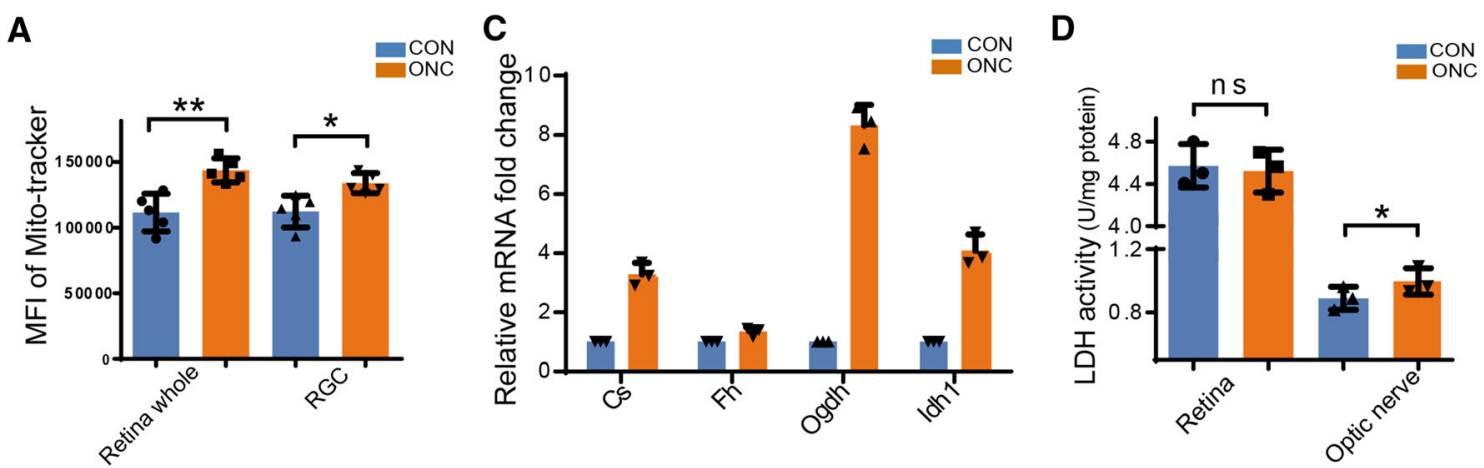

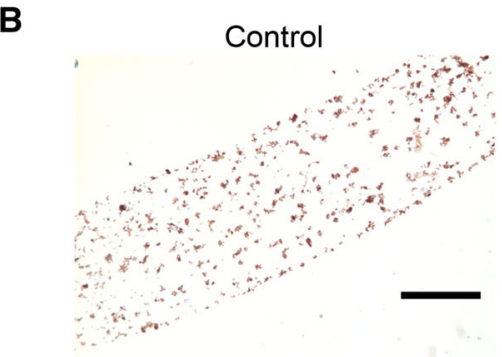

E

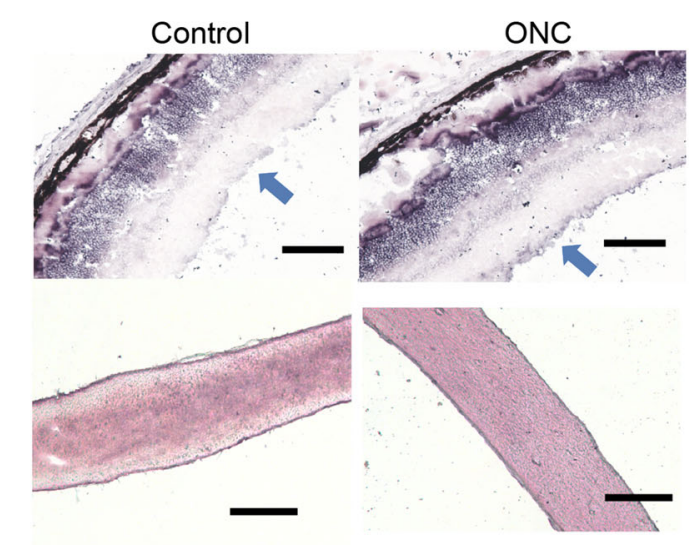

Fig. 2 Changes in glycolysis and mitochondrial oxidative phosphorylation after ONC. A Statistical analysis of the mean fluorescence intensity of MitoTracker staining in retinal cells and RGCs. B Representative images and quantitative analysis of diaminobenzidine staining of cytochrome oxidase in injured and sham-operated optic nerves. C mRNA levels of tricarboxylic acid cycle enzymes in crushed optic nerves after ONC. D Quantitative analysis of lactate

of glycolysis and respiration increased or decreased. The data showed that the ONC/CON value after the CCCP injection $\left(\mathrm{ONC}_{\mathrm{CCCP}} / \mathrm{CON}_{\mathrm{CCCP}}\right)$ was greater than the control $\mathrm{ONC} / \mathrm{CON}$ value, while no significant difference was found between $\mathrm{ONC} / \mathrm{CON}$ and $\mathrm{ONC}_{2 \mathrm{DG}} / \mathrm{CON}_{2 \mathrm{DG}}$ (Fig. 5B). These calculations suggested a greater proportion of oxidative phosphorylation in the injured optic nerve than in the intact nerve. For further validation, additional glycolysis and oxidative phosphorylation inhibitors were applied - iodoacetic acid was used to inactivate glyceraldehyde-3-P dehydrogenase, and oligomycin was used to block membrane-bound mitochondrial ATP synthase and

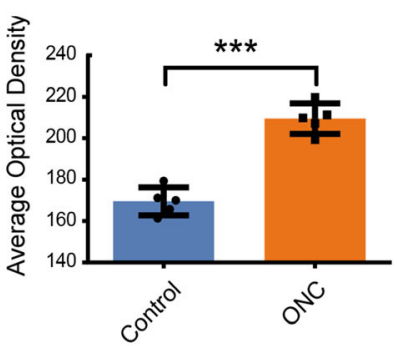

$\mathbf{F}$

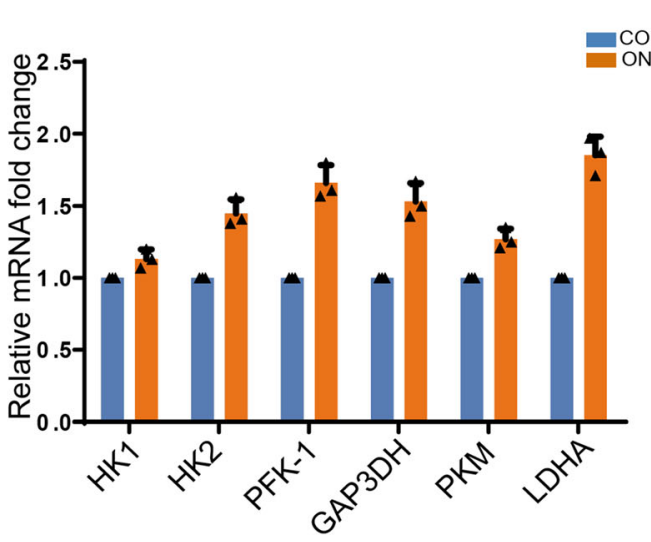

dehydrogenase (LDH) activity in the optic nerve and whole retina after ONC. E Representative images of LDH activity (blue/purple staining) in the retina (upper) and the optic nerve (lower) after injury [note intense and disseminated staining in the RGC layer (blue arrows); qualitative analysis in Table S1]. F mRNA levels of glycolytic enzymes in optic nerves after ONC. Scale bars in B and E, $100 \mu \mathrm{m}$.

proton channels - and they produced similar results (Fig. 5C). Thus, metabolic activation along with a metabolic shift toward respiration occurs in traumatized optic nerves.

The disproportionate increase in respiration caught our attention, raising the question of its biological significance. Is it beneficial or deleterious for RGC preservation? Experiments with PTEN deletion mice helped to provide answers to these questions. PTEN deletion is well established as a general factor controlling neuronal growth, and it has frequently been shown to preserve neurons in the context of CNS injury [40]. Downregulation of PTEN has 


\section{A}
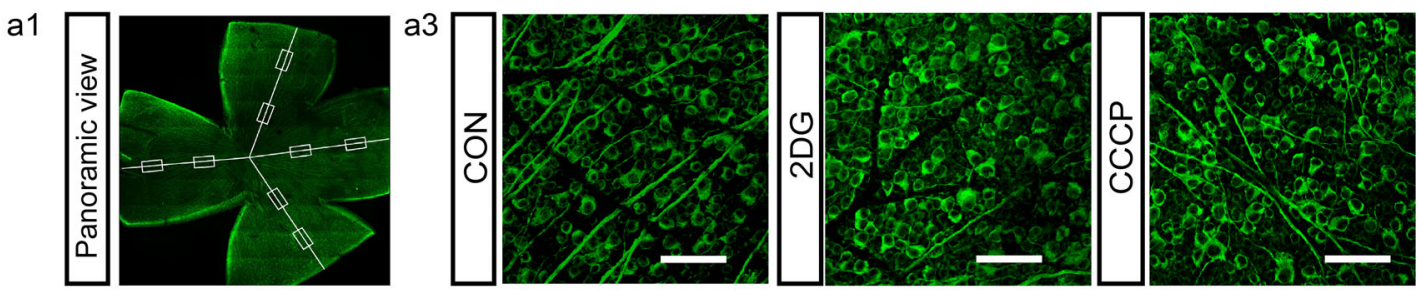

a2
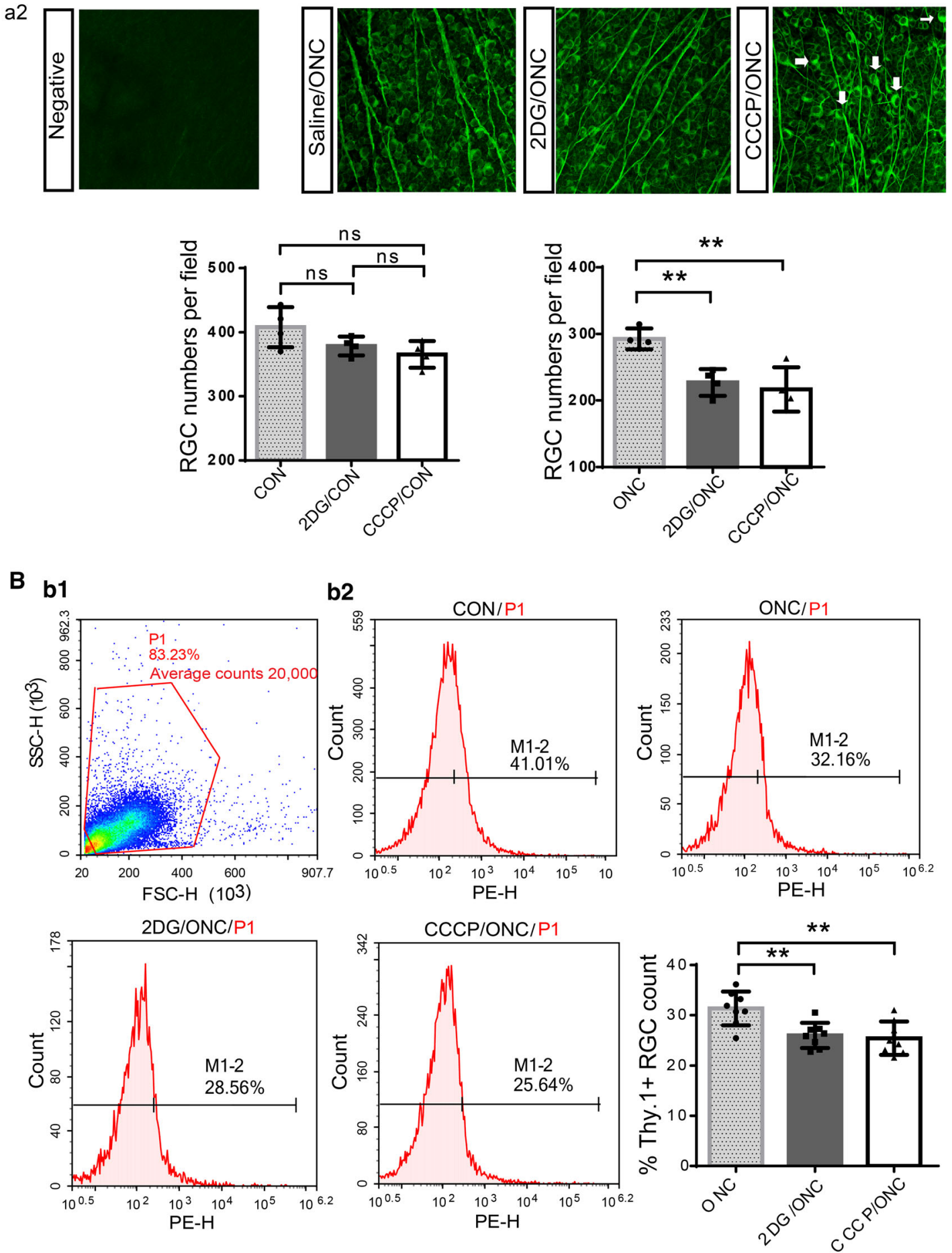
4Fig. 3 Blocking the ATP supply by inhibiting glycolysis or oxidative phosphorylation increases the retinal damage. A Images of flatmounted retinas stained with Tuj1; eight fields from each retinal explant (boxes) were analyzed. a1 Representative example of RGC layers in a whole-mount retina stained for Tuj1 (green). a2 Representative image of the negative control, from which the primary antibody (Tuj1) was omitted. a3 Representative confocal images from the central regions of retinas in each group. The ONC group with carbonyl cyanide 3-chlorophenylhydrazone (CCCP) injection displayed swollen somata with tubulin accumulation (arrows). Scale bars, $200 \mu \mathrm{m}$. B Flow cytometry-based sorting strategy for Thy-1.2positive cells. b1 An initial gated population (P1) was plotted to discard clumped cells or aggregates and obtain a single-cell population. b2 Post-sorting analysis of the percentage of Thy-1.2-positive RGCs (M1-2) in each group (note greater RGC death in the 2DG and CCCP groups 1 day after ONC).

been shown to increase RGC survival in an ONC model [20, 41]. PTEN is also a metabolic regulator that can suppress glycolysis and cause an energetic shift toward mitochondrial respiration when activated [42, 43]. Based on this evidence, we investigated whether respirationbiased metabolism contributes to the limited neuron survival we found and whether PTEN deletion restricts respiration, thus protecting RGCs.

The GSEA results from a microarray (GSE32309) of PTEN-deficient and wild-type mice subjected to ONC supported our hypothesis. The data revealed that the top five pathways enriched in wild-type mice were KEGG_OXIDATIVE_PHOSPHORYLATION, KEGG_RIBOSOME, KEGG_ETHER_LIPID_METABOLISM, KEGG_RNA_POLYMERASE, and KEGG_RETINOL_METABOLISM (Fig. 6A). Although oxidative phosphorylation was ranked first in the wild-type group, it was not enriched in the PTEN-deficient group (Fig. 6B). Thus, a high level of oxidative phosphorylation is a prominent and potentially adverse process occurring in retinal neurons after optic nerve injury.

\section{Redirecting Metabolism toward Glycolysis (War- burg Effect) Protects against RGC Loss}

If the above hypothesis is correct, then restricting oxidative phosphorylation should benefit RGC survival in the ONC model. We tried to increase the activity of another primary energy source, i.e., glycolysis over respiration, similar to the "Warburg effect" in tumors and PTEN-deficient mice, to avoid oxidative phosphorylation overload and simultaneously maintain ATP production. We performed a literature search and identified a drug that shifts energy dependence to glycolysis. Paul S Brookes and Vamsi K Mootha examined $>3,500$ small molecules and discovered that the clinically-used drug meclizine attenuates mitochondrial respiration without reducing mitochondrial biogenesis or viability and augments glycolysis in various models $[44,45]$. We intraperitoneally injected $100 \mathrm{mg} / \mathrm{kg}$ meclizine $17 \mathrm{~h}$ and $3 \mathrm{~h}$ before $\mathrm{ONC}$, as suggested in previous reports [44, 45], and measured the metabolic effects. Although total ATP levels and the ADP/ATP ratio were not significantly altered in injured optic nerves (Fig. 7A, B), LDH staining and activity were markedly increased in meclizine-treated optic nerves (Fig. 7C, D). The contribution of glycolysis to the energy output increased, while the contribution of mitochondrial respiration decreased (Fig. 7E). Thus, meclizine was able to shift the metabolic dependence toward glycolysis rather than mitochondrial respiration.

As the preference for mitochondria-derived ATP production is reduced by activating glycolysis, meclizine achieved a favorable reduction in RGC death (Fig. 8A, B). To confirm that activation of glycolysis is indispensable for this neuroprotective effect, we injected 2DG into the meclizine-protected ONC model and found a mitigation of the positive effect manifested as further RGC loss (Fig. 8A). Therefore, the disproportionate activation of respiration following acute axon injury is a major contributor to RGC damage, and the redirection of energy metabolism toward glycolysis may enhance neuronal survival (Fig. 8B).

\section{Discussion}

To help injured neurons better survive an injury energetically, we must determine their reactions to intense postinjury energy demands [5]. Metabolic plasticity enables cells to adapt to acute energy demands, with which neurons are not typically associated. However, in the present study, the optic nerve and retina displayed metabolic activation 1 day after injury, with signs of increased ATP and ADP levels and enhanced glycolysis and oxidative phosphorylation (Figs 1 and 2). These results differ from the findings of several in vitro studies in which neurons tend to display energy deficits after trauma originating from mitochondrial degeneration $[7,46]$, but they are consistent with an in vivo study showing that injury increases the number of motile mitochondria during the early phase of motor nerve regeneration [47]. This discrepancy may be explained by differences between in vitro and in vivo conditions. In vivo, neurons are supported by the surrounding environment, particularly relying on astrocytes to provide lactate and pyruvate for oxidative phosphorylation [48-50]. Energetic support from activated astrocytes to neurons is important during early neurodegeneration and periods of increased energy demand [51-53], while isolated cultured neurons are typically cultured in $25 \mathrm{mmol} / \mathrm{L}$ glucose, a hyperglycemic environment that may disturb glucose 
A

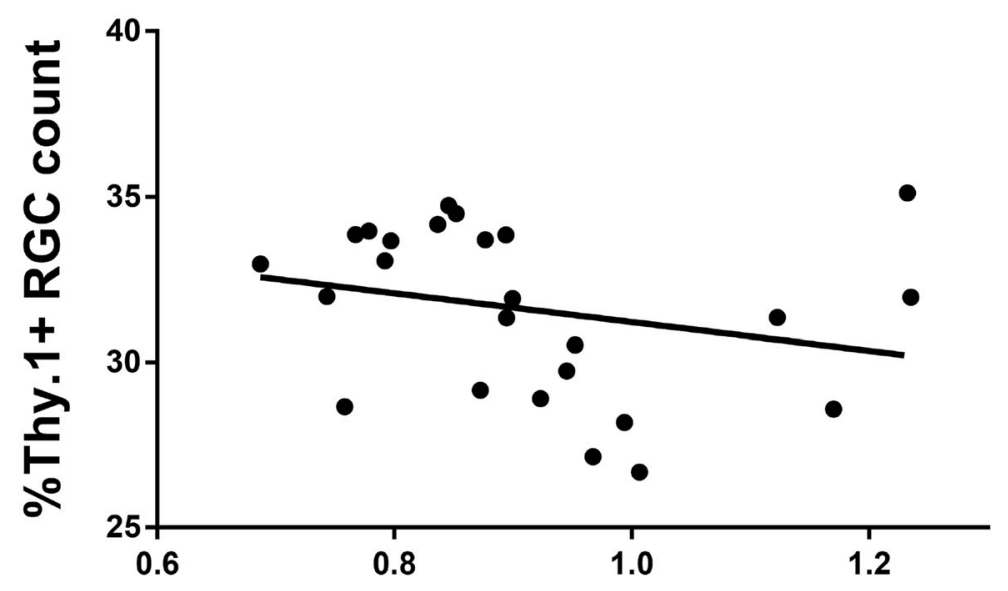

Fold change of ADPIATP after ONC

B

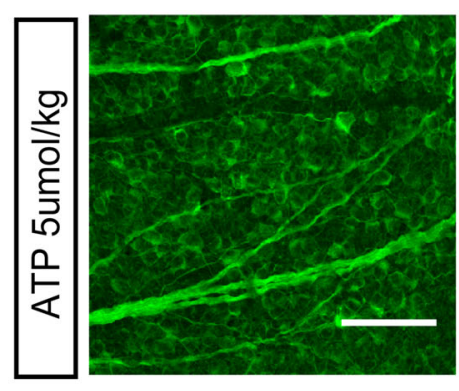

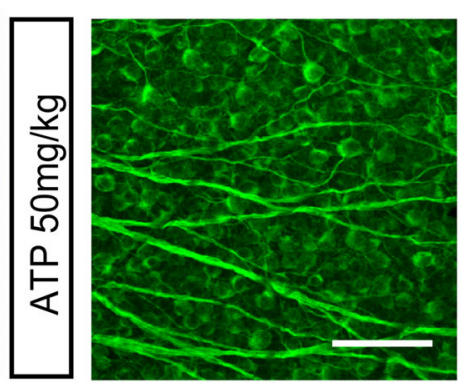

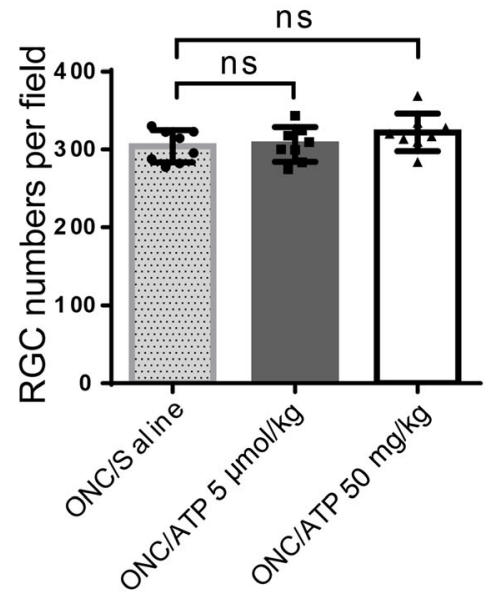

C
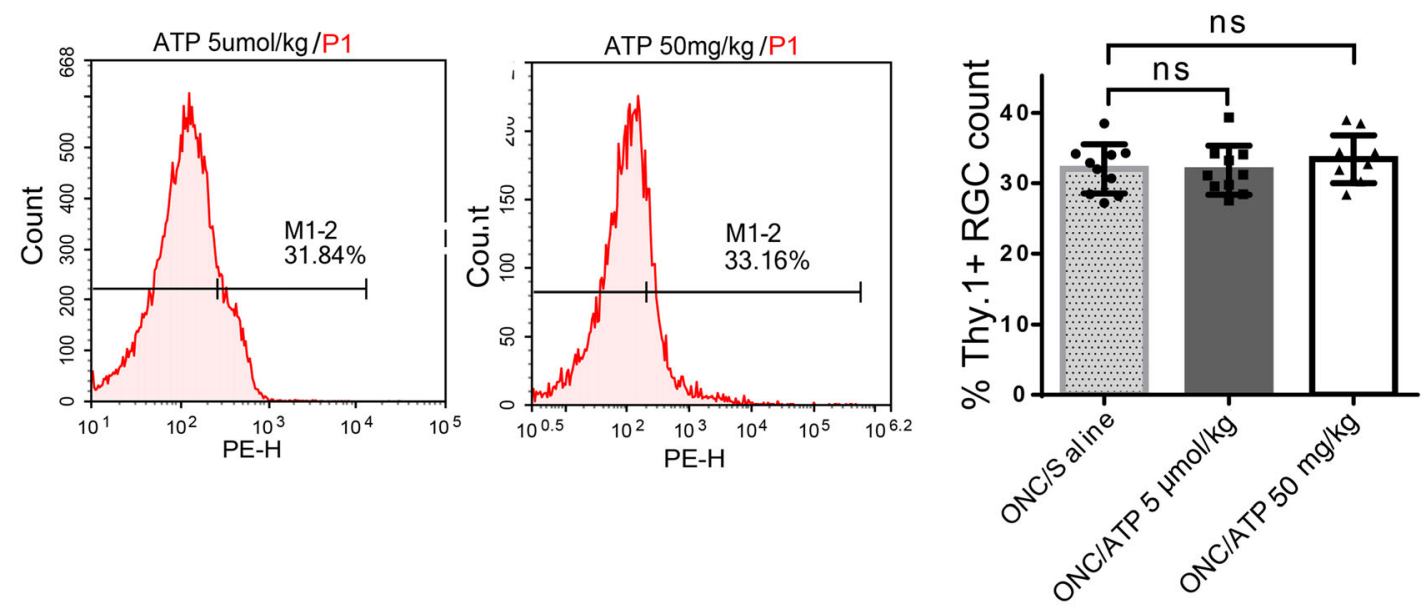

Fig. 4 Neither greater endogenous energy reserves nor exogenous ATP supplementation results in reduced RGC loss. A Pearson's correlation analysis revealed no significant correlation between the numbers of Thy-1.2-positive RGCs and ATP reserves $(n=25 ; r=$ $-0.2507 ; P=0.2268$ ). The line represents the linear regression equation

metabolism, and these neurons rarely survive on their own. The mitochondrial degeneration in vitro may reflect of the data ( $\mathrm{Y}=-4.337 * \mathrm{X}+35.55, R^{2}=0.06283$, not significant), $r$, correlation coefficient. $R^{2}$ is calculated from the multivariate linear regression. B Neither $5 \mu \mathrm{mol} / \mathrm{kg}$ nor $50 \mathrm{mg} / \mathrm{kg}$ ATP injection had an effect on RGC survival after ONC (scale bars, $200 \mu \mathrm{m}$ ). C Percentages of Thy-1.2-positive RGCs with and without ATP injection.

ongoing neuronal death with inactive neuron repair and recovery after damage. Notably, we monitored the energy 
A

$$
\text { From } \frac{\mathrm{ONC}_{20 \mathrm{G}}}{\mathrm{CON}_{2 \mathrm{GG}}}=\frac{\mathrm{ONC}-\mathrm{E}_{20 \mathrm{G} / \mathrm{O}}}{\mathrm{CON}-\mathrm{E}_{20 \mathrm{G} / \mathrm{C}}}
$$

the following equation can be derived:

$$
\begin{aligned}
& \frac{\mathrm{ONC}_{2 D G}}{\mathrm{CON}_{\mathrm{BOG}}}-\frac{\mathrm{ONC}}{\mathrm{CON}}=\frac{\mathrm{ONC}-\mathrm{E}_{2 \mathrm{DG} O}}{\mathrm{CON}-\mathrm{E}_{2 \mathrm{OGC}}}-\frac{\mathrm{ONC}}{\mathrm{CON}} \\
& =\frac{C O N \times O N C-C O N \times E_{20 G 10}-C O N \times O N C+O N C \times E_{20 G / C}}{C O N-E_{2 D G 1 C} \times C O N} \\
& =\frac{O N C \times E_{20 G / C}-C O N \times E_{20 G / 0}}{\left(C O N-E_{20 G / C}\right) \times C O N}
\end{aligned}
$$

Corresponding equation or inequation goes here:

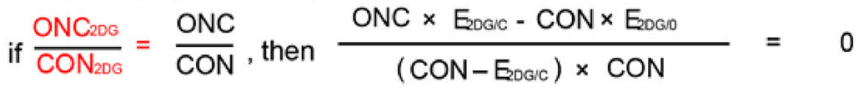

$$
\begin{aligned}
& \mathrm{ONC} \times \mathrm{E}_{2 \mathrm{DGIC}}-\mathrm{CON} \times \mathrm{E}_{2 \mathrm{OG}}=0 \\
& \frac{E_{2 D G / O}}{E_{2 D G / C}}=\frac{O N C}{C O N} \text {, which means that the glycolysis-derived ATP proportion in ONC }
\end{aligned}
$$

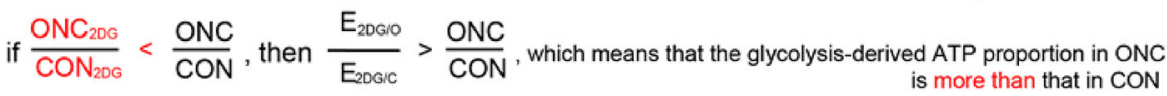

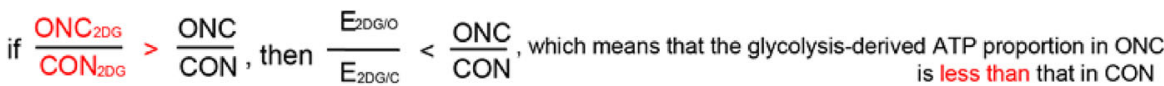

The proportion of respiration-derived ATP can be deduced in the same way.

B

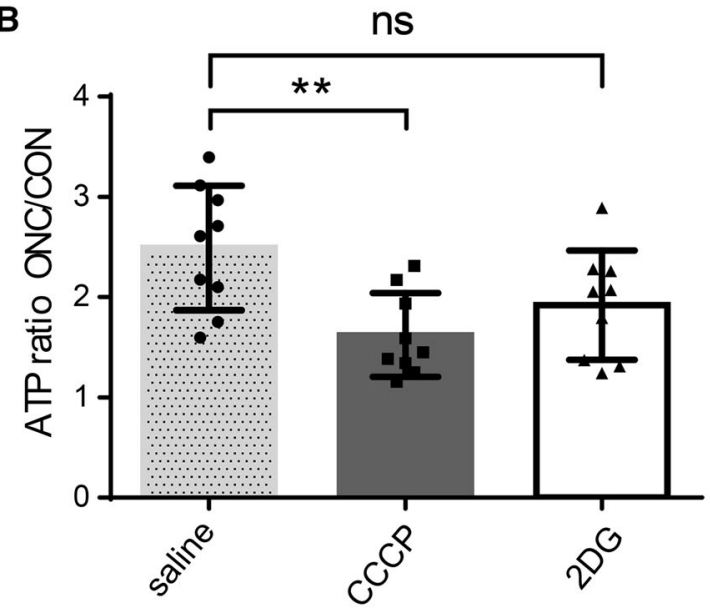

Fig. 5 Energy metabolism shifts to oxidative phosphorylation in injured optic nerve. A Derivation of the formula for measuring the changes in each pathway and their contributions to energy production. The algorithm establishes $\mathrm{ONC}_{2 \mathrm{DG}} / \mathrm{CON}_{2 \mathrm{DG}}$ as an index for evaluating an altered ratio of glycolysis and respiration. B, C Calculated

status at the tissue level (whole retina with a mixture of cells and optic nerve with predominantly RGC axons), because it is technically difficult to separate the metabolism of neurons and glia. Thus, the potential neuronal origin of these metabolic changes remains unclear. Measurements at the cellular level are needed to address this problem in future studies.

Although intense energy production is vital for RGC survival after ONC (Fig. 3), increasing the energy level
C

ns

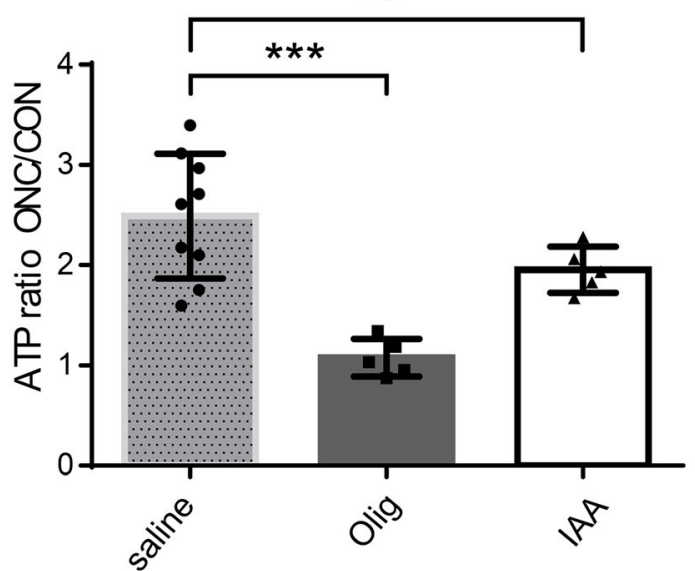

ATP ratios from injured/intact optic nerves. $\mathrm{ONC}_{\mathrm{CCCP}} / \mathrm{CON}_{\mathrm{CCCP}}$ and $\mathrm{ONC}_{\text {oligomycin }} / \mathrm{CON}_{\text {oligomycin }}$ were decreased in traumatized optic nerves (note that the relative contribution of oxidative phosphorylation to the energy supply increased following ONC and the proportion of glycolysis showed no significant change)

largely failed to promote RGC survival. Our results suggested that inherently high energy levels in the injured optic nerve have no significant effect on RGC survival (Fig. 4A), probably because the spontaneous metabolic activation in the optic nerve and retina is sufficient to satisfy most urgent energy demands during early trauma. Energy-boosting neuroprotection has exhibited great potential in several chronic neurodegenerative conditions, such as Huntington's disease and Parkinson's disease [54-56]. 
Fig. 6 Gene set enrichment analysis demonstrating enriched pathways in injured wild-type versus PTEN-deleted retinas. A The top five pathways enriched in injured wild-type mice compared to PTENdeleted mice, with oxidative phosphorylation ranked first. Bar plot, SIZE of the top 5 enriched gene sets; red, enrichment score; green, normalized enrichment score. (ES, the degree to which a gene set is overrepresented at the top or bottom of a ranked list of genes; NES, differences in gene set size and correlations between gene sets and the expression dataset; SIZE, number of genes in the gene set after filtering out those not in the expression dataset). B Enrichment plot of the KEGG_OXIDATIVE_ PHOSPHORYLATION pathway (note that significant enrichment of "oxidative phosphorylation" genes was found in injured retinal cells from wild-type mice compared with those from PTEN deletion mice).
A

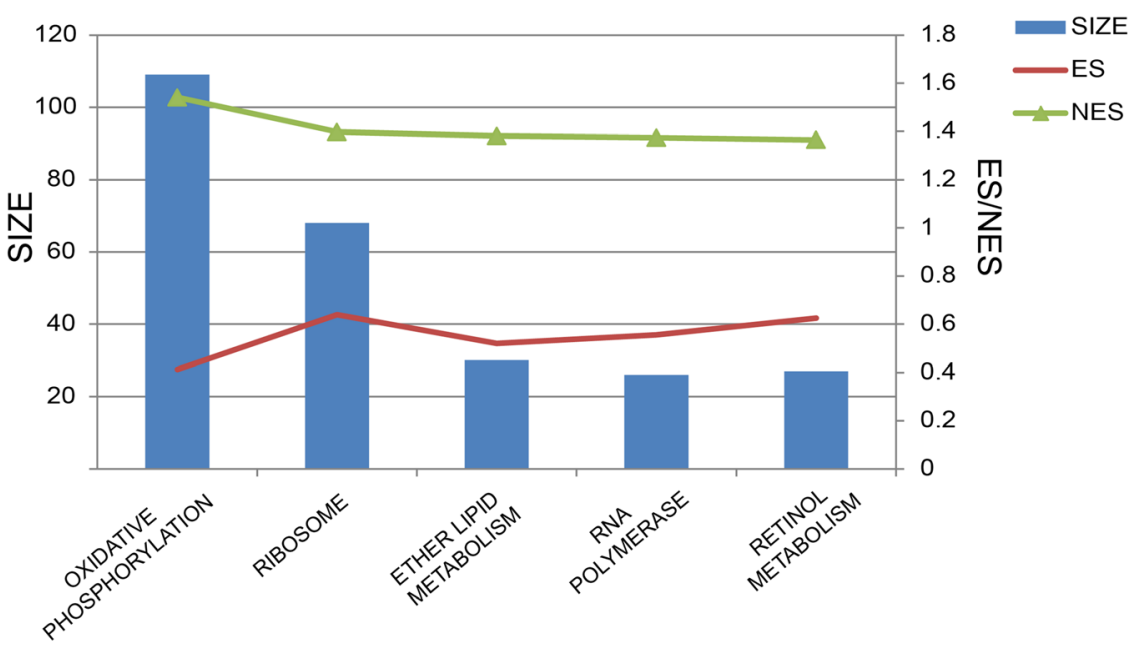

B

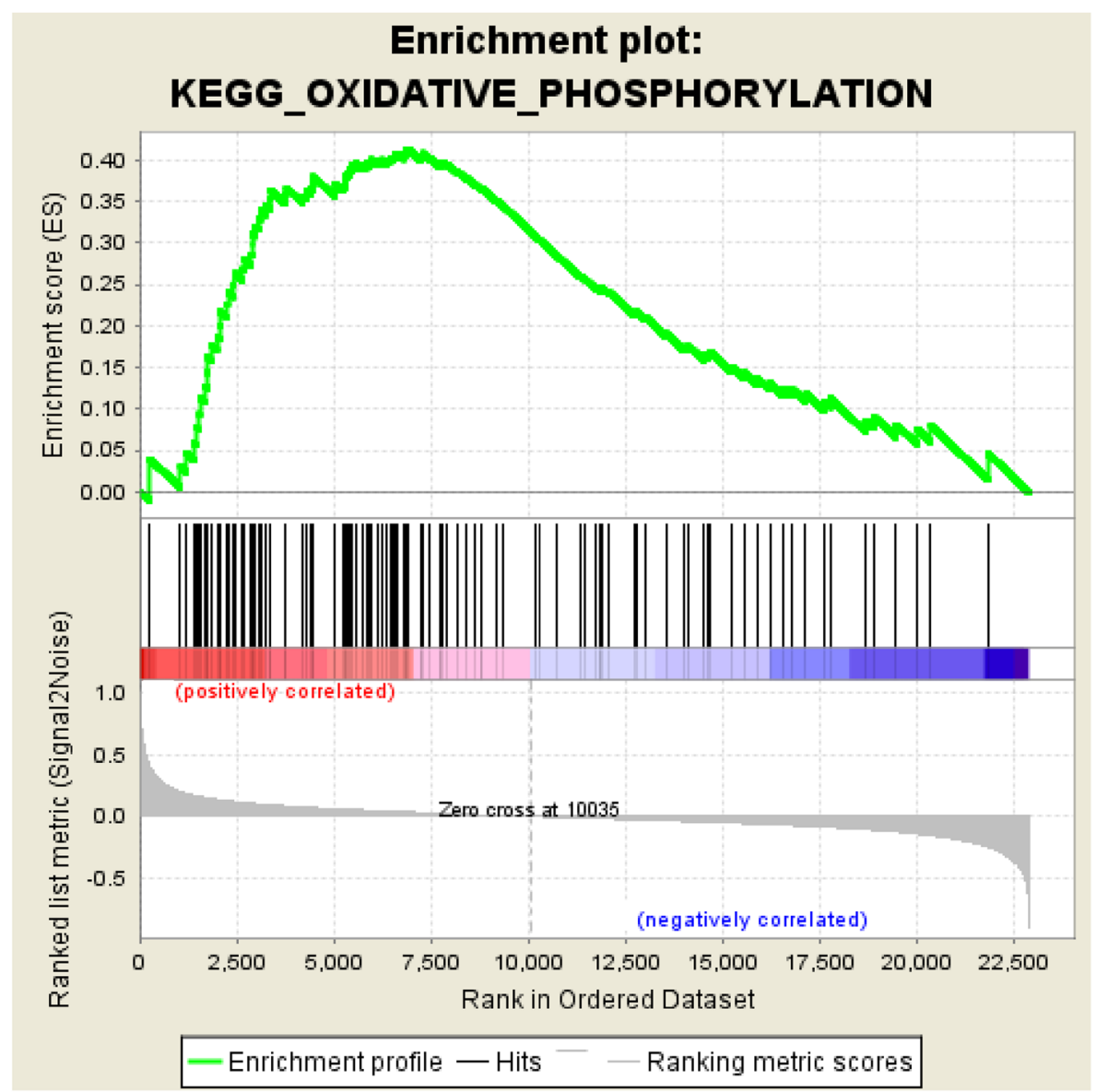

However, during acute clinical neuronal injury, such as stroke, this approach is ineffective or even detrimental [57], similar to our acute ONC model. The timing of treatment during the progression of neuronal demise may be a factor. Our results revealed that the increased ATP levels gradually declined over time (1, 3, and 7 days) in the ONC model (Fig. 1F), indicating that the activated energetic response may be an acute adaptation during the early stage of trauma and that energy exhaustion may occur in the later stage of acute neuronal trauma, thus mimicking a chronic neurodegenerative state. Evidence related to glaucoma, a chronic optic nerve injury, also supports our suspicion. RGCs from DBA/2J mice with early-stage glaucoma show higher expression of oxidative phosphorylation genes than those from healthy mice [58]; however, mice with later-stage chronic glaucoma display 

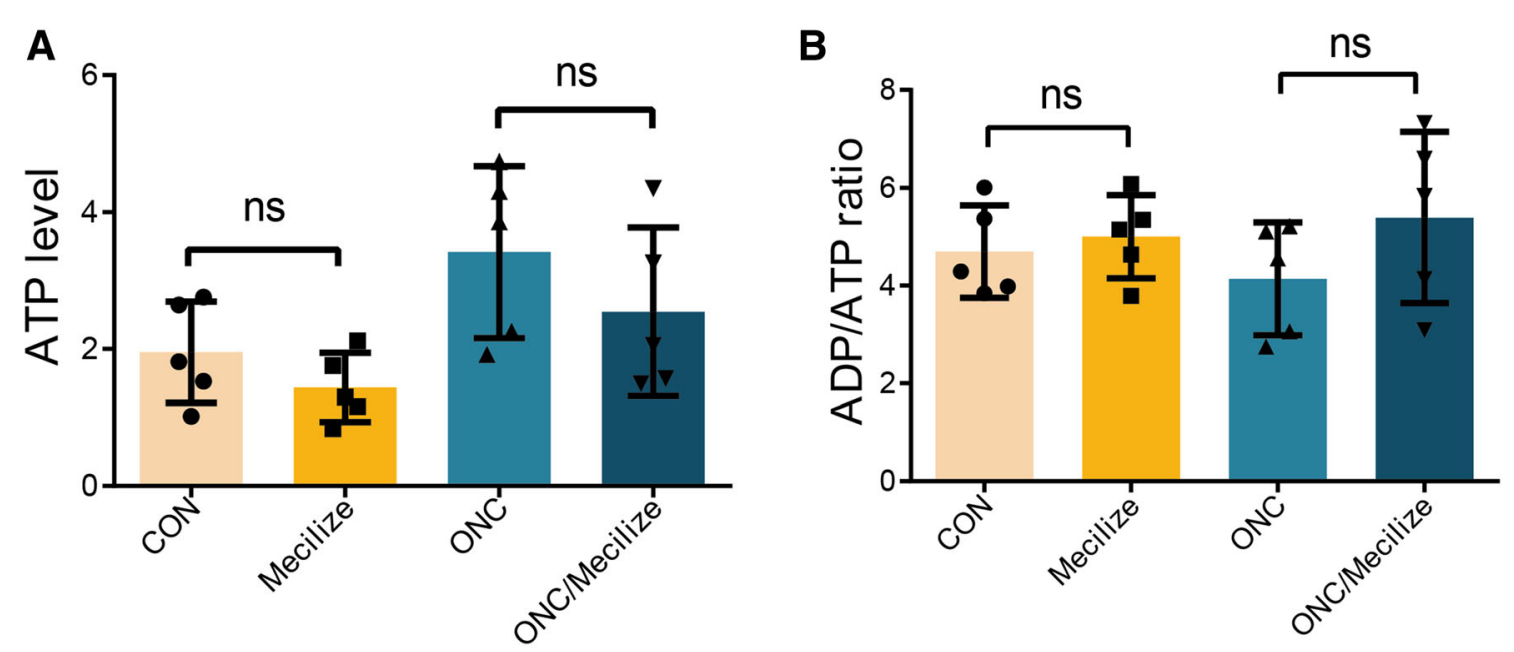
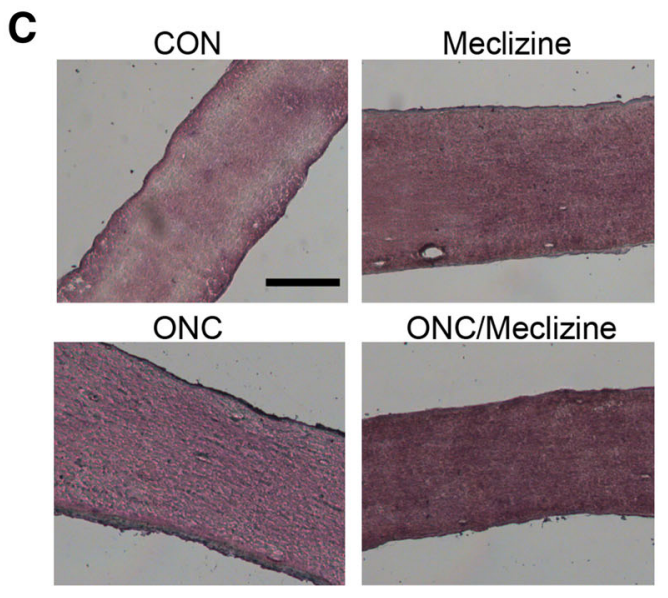

E

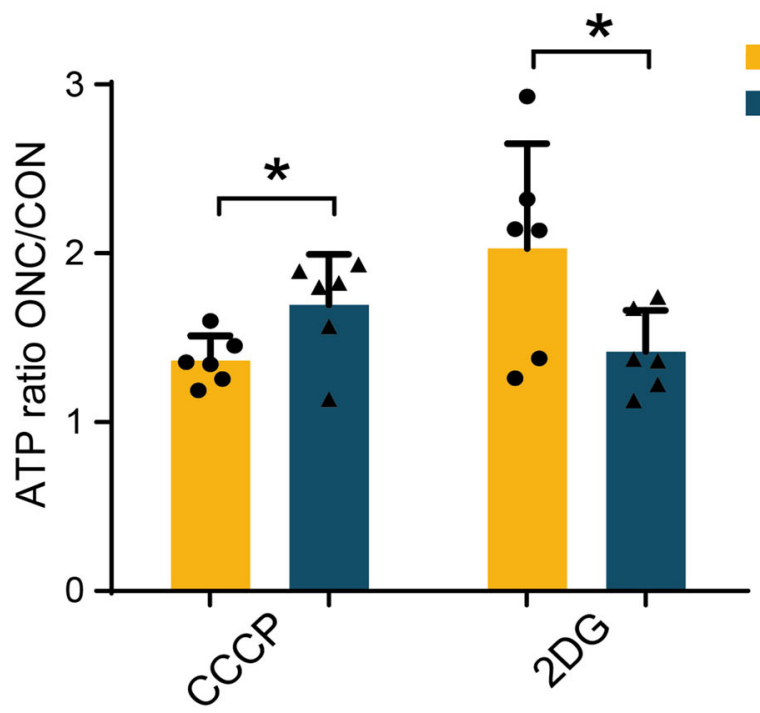

D

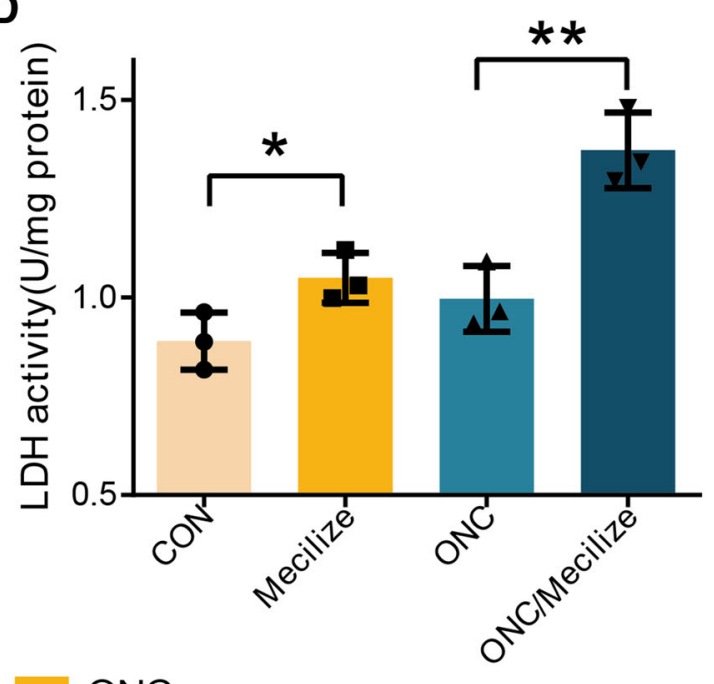

ONC

ONC/Meclizine
Fig. 7 Meclizine redirects metabolic flux toward glycolysis. A, B Pretreatment with meclizine did not significantly change ATP levels (A) or ADP/ATP ratios (B). C Representative images of LDH staining (note the stronger staining in the meclizine group than in the control group, and in the meclizine pretreatment ONC group than in the ONC group; histochemical ratings in Table S2). Scale bar, 100 $\mu \mathrm{m}$. D LDH activity in the meclizine treatment and the $\mathrm{ONC} /$ meclizine groups. $\mathbf{E}$ Effects of pretreatment with meclizine on $\mathrm{ONC}_{\mathrm{CCCP}} / \mathrm{CON}_{\mathrm{CCCP}}$ and $\mathrm{ONC}_{2 \mathrm{DG}} / \mathrm{CON}_{2 \mathrm{DG}}$ in injured optic nerves. 
A

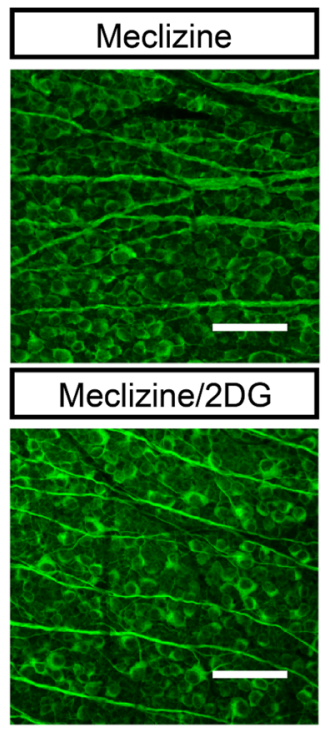

B

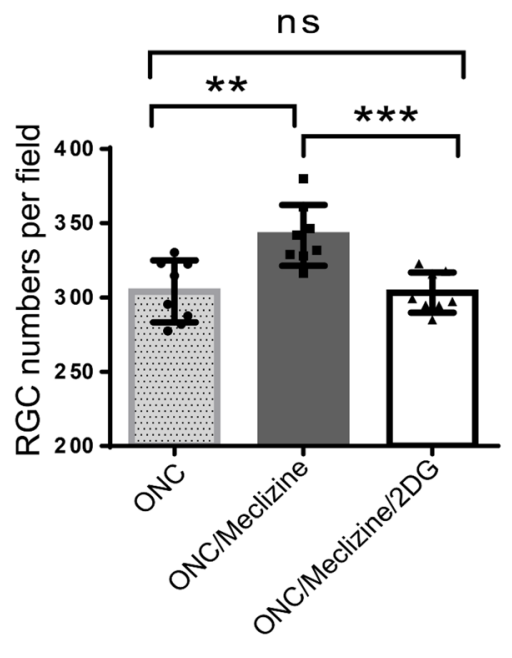

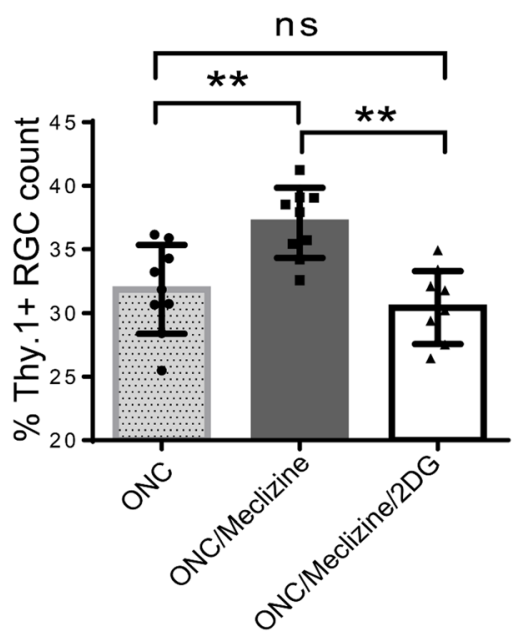

C

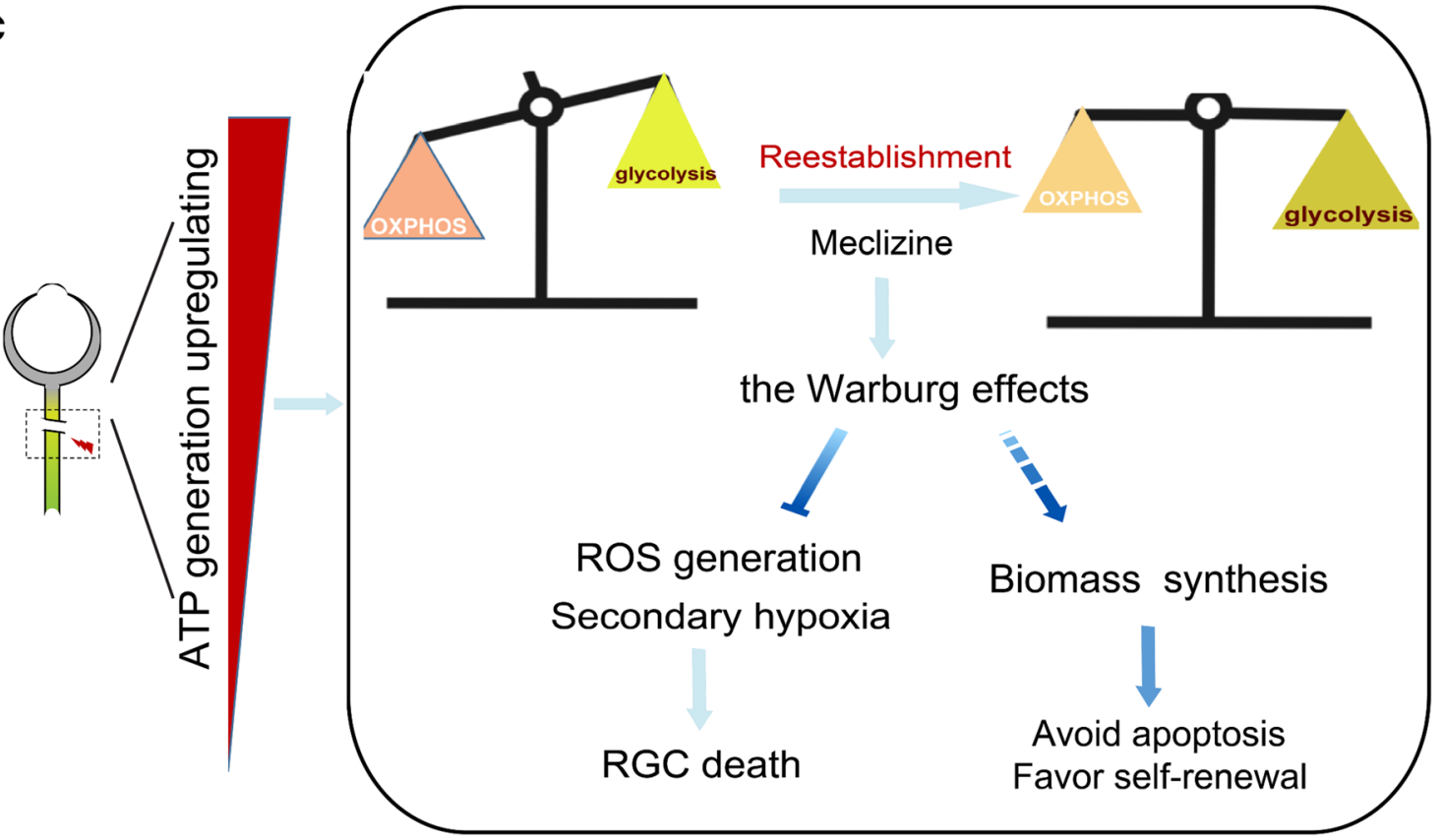

Fig. 8 Meclizine pretreatment protects RGCs from death, and this is reversed by 2 DG. A Meclizine pretreatment prevents RGC death, and 2DG supplementation diminishes this effect. Scale bars, $200 \mu \mathrm{m}$. Representative images of whole-mount retina stained for Tuj1 in Meclizine-treated group and Meclizine/2DG-treated group. B RGC numbers based on flow cytometry (note that they are consistent with the findings from retinal whole mounts). C A working model of metabolic redirection toward glycolysis (Warburg effect) to benefit

mitochondrial dysfunction and energy compromise [59], and patients with advanced primary open-angle glaucoma suffer from oxidative phosphorylation complex-I damage and impaired ATP synthesis [60, 61].

We found increased glycolysis and oxidative phosphorylation and that oxidative phosphorylation temporarily
RGC survival. Disproportionate upregulation of oxidative phosphorylation over glycolysis after ONC may confer several vulnerabilities for RGC survival, including reactive oxygen species (ROS) accumulation and secondary hypoxia. However, re-establishing energy homeostasis by restoring the preference for glycolysis is hypothesized to provide survival advantages to the cells, such as favoring macromolecule biosynthesis and promoting self-renewal.

outperforms other pathways, providing a stronger response to the increased demand (Fig. 5). The relative contributions of glycolytic flux and oxidative phosphorylation under normal conditions or in response to changing energy requirements in the CNS have not been previously described, potentially because of the lack of available 
measurement tools and sensitive assays. Our conclusion was first drawn according to calculations using formulas. However, statistical analysis cannot provide an accurate value for the percentage increase or decrease. As more advanced techniques emerge, these proportions should be precisely measured.

Our data showed that prominent activation of oxidative phosphorylation was associated with a poor prognosis for RGC survival (Fig. 6), as suggested by comparisons between PTEN conditional knockout mice and wild-type mice. Meclizine is an 'older' FDA-approved compound that has been shown in a drug screen and multiple animal models to be capable of reprogramming energy metabolism [44]. Our study found that, by redirecting metabolism toward glycolysis using meclizine, RGC survival was promoted (Fig. 8A, B). Why is the pharmacological augmentation of glycolysis protective? The underlying causes may be similar in our model and in the "Warburg effect" in tumors (Fig. 8) [62-64]. Overactive oxidative phosphorylation can cause extensive ROS production, and the resultant oxidative stress has been confirmed to be a major cause of RGC apoptosis after axon injury [65-67]. In addition, respiratory substrate influx into mitochondria may cause sensitivity to mitochondria-mediated apoptosis [68-70], which may also lead to excessive respirationinduced RGC loss in the ONC model. Furthermore, a metabolic preference for glycolysis is an inherent trait of neural progenitor cells [71, 72], which may indicate neural stem cell activation in our model, potentially representing an additional step toward neuronal protection and regeneration. In conclusion, we provided clues that pathologically augmented mitochondrial respiration may be a double-edged sword in the injured optic nerve. That is, excessive activation of mitochondrial respiration causes massive secondary damage to RGCs despite producing energy (Fig. 8C). This model can also explain why increased internal energy generation is not significantly correlated with RGC survival, as illustrated in Fig. 3. Exactly how these imbalances in the reallocation of energy production pathways lead to neuropathology warrants further investigation. Overall, as a first step toward elucidating how neuronal energy metabolism adapts to axonal insults in vivo, our study puts forward a potential bioenergetic strategy to facilitate neuronal survival in the traumatized CNS that involves shifting energy metabolism toward glycolysis (the Warburg effect).

Acknowledgements The authors are grateful to Dr. Sen Lin, Professor Jun Yan, and Professor Feng Mei from the Third Military Medical University for their advice regarding the research design. This work was supported by the National Natural Science Foundation of China (81371006). The funders had no role in the study design, data collection, data analysis, or interpretation of the work.
Conflict of Interest The authors have no conflicts of interest to declare.

Open Access This article is licensed under a Creative Commons Attribution 4.0 International License, which permits use, sharing, adaptation, distribution and reproduction in any medium or format, as long as you give appropriate credit to the original author(s) and the source, provide a link to the Creative Commons licence, and indicate if changes were made. The images or other third party material in this article are included in the article's Creative Commons licence, unless indicated otherwise in a credit line to the material. If material is not included in the article's Creative Commons licence and your intended use is not permitted by statutory regulation or exceeds the permitted use, you will need to obtain permission directly from the copyright holder. To view a copy of this licence, visit http://creativecommons. org/licenses/by/4.0/.

\section{References}

1. Volpicelli-Daley L, Luk K, Patel T, Tanik S, Riddle D, Stieber A, et al. Exogenous $\alpha$-synuclein fibrils induce Lewy body pathology leading to synaptic dysfunction and neuron death. Neuron 2011, 72: $57-71$.

2. Frakes A, Ferraiuolo L, Haidet-Phillips A, Schmelzer L, Braun L, Miranda $\mathrm{C}$, et al. Microglia induce motor neuron death via the classical NF- $\kappa \mathrm{B}$ pathway in amyotrophic lateral sclerosis. Neuron 2014, 81: 1009-1023.

3. Belanger M, Allaman I, Magistretti PJ. Brain energy metabolism: focus on astrocyte-neuron metabolic cooperation. Cell Metab 2011, 14: 724-738.

4. Mink JW, Blumenschine RJ, Adams DB. Ratio of central nervous system to body metabolism in vertebrates: its constancy and functional basis. Am J Physiol 1981, 241: R203-212.

5. Bradke F, Fawcett JW, Spira ME. Assembly of a new growth cone after axotomy: the precursor to axon regeneration. Nat Rev Neurosci 2012, 13: 183-193.

6. Kiryu-Seo S, Ohno N, Kidd G, Komuro H, Trapp B. Demyelination increases axonal stationary mitochondrial size and the speed of axonal mitochondrial transport. J Neurosci 2010, 30: 6658-6666.

7. Yokota S, Takihara Y, Arimura S, Miyake S, Takamura Y, Yoshimura $\mathrm{N}$, et al. Altered transport velocity of axonal mitochondria in retinal ganglion cells after laser-induced axonal injury in vitro. Invest Ophthalmol Vis Sci 2015, 56: 8019-8025.

8. White H, Venkatesh B. Clinical review: Ketones and brain injury. Critical Care 2011, 15: 219-219.

9. Yellen G. Fueling thought: Management of glycolysis and oxidative phosphorylation in neuronal metabolism. J Cell Biol 2018, 217: 2235-2246.

10. Pfeiffer T, Schuster S, Bonhoeffer S. Cooperation and competition in the evolution of ATP-producing pathways. Science 2001, 292: 504-507.

11. Warburg O, Wind F, Negelein E. The metabolism of tumors in the body. J Gen Physiol 1927, 8: 519-530.

12. Winkler BS. Glycolytic and oxidative metabolism in relation to retinal function. J Gen Physiol 1981, 77: 667-692.

13. Winkler BS, Starnes CA, Twardy BS, Brault D, Taylor RC. Nuclear magnetic resonance and biochemical measurements of glucose utilization in the cone-dominant ground squirrel retina. Invest Ophthalmol Vis Sci 2008, 49: 4613-4619.

14. House SW, Burk D, Schade AL. On respiratory impairment in cancer cells. Science 1956, 124: 267-272. 
15. Magharious MM, D’Onofrio PM, Koeberle PD. Optic nerve transection: a model of adult neuron apoptosis in the central nervous system. J Vis Exp 2011, 51: e2241.

16. Huntwork-Rodriguez S, Wang B, Watkins T, Ghosh A, Pozniak $\mathrm{C}$, Bustos D, et al. JNK-mediated phosphorylation of DLK suppresses its ubiquitination to promote neuronal apoptosis. J Cell Biol 2013, 202: 747-763.

17. Watkins T, Wang B, Huntwork-Rodriguez S, Yang J, Jiang Z, Eastham-Anderson J, et al. DLK initiates a transcriptional program that couples apoptotic and regenerative responses to axonal injury. Proc Natl Acad Sci U S A 2013, 110: 4039-4044.

18. Mac Nair CE, Schlamp CL, Montgomery AD, Shestopalov VI, Nickells RW. Retinal glial responses to optic nerve crush are attenuated in Bax-deficient mice and modulated by purinergic signaling pathways. J Neuroinflammation 2016, 13: 93.

19. Gkotsi D, Begum R, Salt T, Lascaratos G, Hogg C, Chau K, et al. Recharging mitochondrial batteries in old eyes. Near infra-red increases ATP. Exp Eye Res 2014, 122: 50-53.

20. Park KK, Liu K, Hu Y, Smith PD, Wang C, Cai B, et al. Promoting axon regeneration in the adult CNS by modulation of the PTEN/mTOR pathway. Science 2008, 322: 963-966.

21. Smith PD, Sun F, Park KK, Cai B, Wang C, Kuwako K, et al. SOCS3 deletion promotes optic nerve regeneration in vivo. Neuron 2009, 64: 617-623.

22. Chintalapudi SR, Djenderedjian L, Stiemke AB, Steinle JJ, Jablonski MM, Morales-Tirado VM. Isolation and molecular profiling of primary mouse retinal ganglion cells: Comparison of phenotypes from healthy and glaucomatous retinas. Front Aging Neurosci 2016, 8, 93. https://doi.org/10.3389/fnagi.2016.00093.

23. Chintalapudi SR, Patel NN, Goldsmith ZK, Djenderedjian L, Wang XD, Marion TN, et al. Isolation of primary murine retinal ganglion cells (RGCs) by flow cytometry. J Vis Exp 2017, 125. https://doi.org/10.3791/55785.

24. Mootha VK, Lindgren CM, Eriksson KF, Subramanian A, Sihag $\mathrm{S}$, Lehar J, et al. PGC-1 $\alpha$-responsive genes involved in oxidative phosphorylation are coordinately downregulated in human diabetes. Nat Genetics 2003, 34: 267.

25. Subramanian A, Tamayo P, Mootha VK, Mukherjee S, Ebert BL, Gillette MA, et al. Gene set enrichment analysis: A knowledgebased approach for interpreting genome-wide expression profiles. Proc Natl Acad Sci U S A 2005, 102: 15545-15550.

26. Edgar R, Domrachev M, Lash AE. Gene Expression Omnibus: NCBI gene expression and hybridization array data repository. Nucleic Acids Res 2002, 30: 207-210.

27. Sun F, Park KK, Belin S, Wang D, Lu T, Chen G, et al. Sustained axon regeneration induced by co-deletion of PTEN and SOCS3. Nature 2011, 480: 372-375.

28. Bolstad BM, Gautier L, Cope L, Irizarry RA. Affy-analysis of Affymetrix GeneChip data at the probe level. Bioinformatics 2004, 20: 307-315.

29. Maldonado EN, Lemasters JJ. ATP/ADP ratio, the missed connection between mitochondria and the Warburg effect. Mitochondrion 2014, 19 Pt A: 78-84.

30. Mor I, Cheung E, Vousden K. Control of glycolysis through regulation of PFK1: old friends and recent additions. Cold Spring Harb Symp Quant Biol 2011, 76: 211-216.

31. Giedrius K, Oglesby EN, Zack DJ, Cone FE, Steinhart MR, Jing $\mathrm{T}$, et al. Retinal ganglion cell morphology after optic nerve crush and experimental glaucoma. Invest Ophthalmol Vis Sci 2012, 90 : 3847-3857.

32. Kayama M, Omura K, Murakami Y, Reshef E, Thanos A, Morizane Y, et al. Combined inhibition of apoptosis and necrosis promotes transient neuroprotection of retinal ganglion cells and partial axon regeneration after optic nerve damage. bioRxiv 357566. https://doi.org/10.1101/357566.
33. Niven J, Laughlin S. Energy limitation as a selective pressure on the evolution of sensory systems. J Exp Biol 2008, 211: 1792-1804.

34. Metallo CM, Vander Heiden MG. Understanding metabolic regulation and its influence on cell physiology. Mol Cell 2013, 49: $388-398$.

35. Cartoni R, Norsworthy MW, Bei F, Wang C, Li S, Zhang Y, et al. The mammalian-specific protein Armcx1 regulates mitochondrial transport during axon regeneration. Neuron 2016, 92: 1294-1307.

36. Wu D, Lee S, Luo J, Xia H, Gushchina S, Richardson PM, et al. Intraneural injection of ATP stimulates regeneration of primary sensory axons in the spinal cord. J Neurosci 2018, 38: 1351-1365.

37. Ren H, Teng Y, Tan B, Zhang X, Jiang W, Liu M, et al. Toll-like receptor-triggered calcium mobilization protects mice against bacterial infection through extracellular ATP release. Infect Immun 2014, 82: 5076-5085.

38. Xiang Y, Wang X, Yan C, Gao Q, Li SA, Liu J, et al. Adenosine5 '-triphosphate (ATP) protects mice against bacterial infection by activation of the NLRP3 inflammasome. PLoS One 2013, 8: e63759.

39. Stachon P, Geis S, Peikert A, Heidenreich A, Michel NA, Unal F, et al. Extracellular ATP induces vascular inflammation and atherosclerosis via purinergic receptor $\mathrm{Y} 2$ in mice. Arterioscler Thromb Vasc Biol 2016, 36: 1577-1586.

40. Knafo S, Esteban JA. PTEN: Local and global modulation of neuronal function in health and disease. Trends Neurosci 2017, 40: 83-91.

41. Shabanzadeh AP, D’Onofrio PM, Magharious M, Choi KAB, Monnier PP, Koeberle PD. Modifying PTEN recruitment promotes neuron survival, regeneration, and functional recovery after CNS injury. Cell Death Dis 2019, 10: 567.

42. Qian X, Li X, Shi Z, Xia Y, Cai Q, Xu D, et al. PTEN suppresses glycolysis by dephosphorylating and inhibiting autophosphorylated PGK1. Mol Cell 2019, 76: 516-527.E7.

43. Garcia-Cao I, Song MS, Hobbs RM, Laurent G, Giorgi C, de Boer VC, et al. Systemic elevation of PTEN induces a tumorsuppressive metabolic state. Cell 2012, 149: 49-62.

44. Gohil VM, Sheth SA, Nilsson R, Wojtovich AP, Lee JH, Perocchi $\mathrm{F}$, et al. Nutrient-sensitized screening for drugs that shift energy metabolism from mitochondrial respiration to glycolysis. Nat Biotechnol 2010, 28: 249-255.

45. Hong CT, Chau KY, Schapira AHV. Meclizine-induced enhanced glycolysis is neuroprotective in Parkinson disease cell models. Sci Rep 2016, 6: 25344.

46. Zhou B, Yu P, Lin MY, Sun T, Chen Y, Sheng ZH. Facilitation of axon regeneration by enhancing mitochondrial transport and rescuing energy deficits. J Cell Biol 2016, 214: 103-119.

47. Han SM, Baig HS, Hammarlund M. Mitochondria localize to injured axons to support regeneration. Neuron 2016, 92: 1308-1323.

48. Philips T, Rothstein J. Oligodendroglia: metabolic supporters of neurons. J Clin Invest 2017, 127: 3271-3280.

49. Bélanger M, Allaman I, Magistretti P. Brain energy metabolism: focus on astrocyte-neuron metabolic cooperation. Cell Metab 2011, 14: 724-738.

50. Zhang Z, Chen W, Zhao Y, Yang Y. Spatiotemporal imaging of cellular energy metabolism with genetically-encoded fluorescent sensors in brain. Neurosci Bull 2018, 34: 875-886.

51. Kuter K, Olech L, Glowacka U, Paleczna M. Astrocyte support is important for the compensatory potential of the nigrostriatal system neurons during early neurodegeneration. J Neurochem 2018, 148: 63-79.

52. Coggan J, Keller D, Calì C, Lehväslaiho H, Markram H, Schürmann F, et al. Norepinephrine stimulates glycogenolysis in 
astrocytes to fuel neurons with lactate. PLoS Comput Biol 2018, 14: e1006392.

53. Sun D, Moore S, Jakobs TC. Optic nerve astrocyte reactivity protects function in experimental glaucoma and other nerve injuries. J Exp Med 2017, 214: 1411-1430.

54. Weinrich TW, Coyne A, Salt TE, Hogg C, Jeffery G. Improving mitochondrial function significantly reduces metabolic, visual, motor and cognitive decline in aged Drosophila melanogaster. Neurobiol Aging 2017, 60: 34-43.

55. Nakano M, Imamura $H$, Sasaoka N, Yamamoto M, Uemura N, Shudo T, et al. ATP maintenance via two types of ATP regulators mitigates pathological phenotypes in mouse models of Parkinson's disease. EBioMedicine 2017, 22: 225-241.

56. Lee J, Kosaras B, Del Signore S, Cormier K, McKee A, Ratan R, et al. Modulation of lipid peroxidation and mitochondrial function improves neuropathology in Huntington's disease mice. Acta Neuropathol 2011, 121: 487-498.

57. Zhang M, Li W, Niu G, Leak R, Chen J, Zhang F. ATP induces mild hypothermia in rats but has a strikingly detrimental impact on focal cerebral ischemia. J Cereb Blood Flow Metab 2013, 33 : e1-e10.

58. Williams PA, Harder JM, Foxworth NE, Cochran KE, Philip VM, Porciatti V, et al. Vitamin B3 modulates mitochondrial vulnerability and prevents glaucoma in aged mice. Science 2017, 355 : 756-760.

59. Harun-Or-Rashid M, Pappenhagen N, Palmer PG, Smith MA, Gevorgyan V, Wilson GN, et al. Structural and functional rescue of chronic metabolically stressed optic nerves through respiration. J Neurosci 2018, 38: 5122-5139.

60. Van Bergen NJ, Crowston JG, Craig JE, Burdon KP, Kearns LS, Sharma S, et al. Measurement of systemic mitochondrial function in advanced primary open-angle glaucoma and leber hereditary optic neuropathy. PLoS One 2015, 10: e0140919.

61. Lee S, Leo S, Crowston JG, Van Bergen NJ, Van, O'Neill EC, O'Hare F, et al. Impaired complex-I-linked respiration and ATP synthesis in primary open-angle glaucoma patient lymphoblasts. Invest Ophthalmol Vis Sci 2012, 53: 2431-2437.
62. Chen X, Qian Y, Wu S. The Warburg effect: evolving interpretations of an established concept. Free Radic Biol Med 2015, 79: 253-263.

63. Liberti MV, Locasale JW. The Warburg effect: how does it benefit cancer cells? Trends Biochem Sci 2016, 41: 211-218.

64. Dasgupta S, Rajapakshe K, Zhu B, Nikolai BC, Yi P, Putluri N, et al. Metabolic enzyme PFKFB4 activates transcriptional coactivator SRC-3 to drive breast cancer. Nature 2018, 556: 249-254.

65. Ott M, Gogvadze V, Orrenius S, Zhivotovsky B. Mitochondria, oxidative stress and cell death. Apoptosis 2007, 12: 913-922.

66. Akiyama G, Azuchi Y, Guo X, Noro T, Kimura A, Harada C, et al. Edaravone prevents retinal degeneration in adult mice following optic nerve injury. Invest Ophthalmol Vis Sci 2017, 58: 4908-4914.

67. Wang DY, Arjun R, Kathryn R, Ceren E, Hyman BT, Wei H, et al. Global gene expression changes in rat retinal ganglion cells in experimental glaucoma. Invest Ophthalmol Vis Sci 2010, 51: 4084-4095.

68. Jeong D, Kim T, Cho I, Kim I. Modification of glycolysis affects cell sensitivity to apoptosis induced by oxidative stress and mediated by mitochondria. Biochem Biophys Res Commun 2004, 313: 984-991.

69. Borutaite V, Budriunaite A, Morkuniene R, Brown GC. Release of mitochondrial cytochrome $\mathrm{c}$ and activation of cytosolic caspases induced by myocardial ischaemia. Biochim Biophys Acta 2001, 1537: 101-109.

70. Shawgo ME, Shelton SN, Robertson JD. Caspase-mediated Bak activation and cytochrome $\mathrm{c}$ release during intrinsic apoptotic cell death in Jurkat cells. J Biol Chem 2008, 283: 35532-35538.

71. Agathocleous M, Love NK, Randlett O, Harris JJ, Liu J, Murray AJ, et al. Metabolic differentiation in the embryonic retina. Nat Cell Biol 2012, 14: 859-864.

72. Tokuda K, Kuramitsu Y, Baron B, Kitagawa T, Tokuda N, Kobayashi M, et al. Changes in metabolic proteins in ex vivo rat retina during glutamate-induced neural progenitor cell induction. Mol Cell Biochem 2016, 419: 177-184. 